\title{
ON APPROXIMATION OF CONTINUOUS FUNCTIONS BY ENTIRE FUNCTIONS OF EXPONENTIAL TYPE ON SUBSETS OF THE REAL LINE
}

\author{
Vladimir V. Andrievskii*
}

\begin{abstract}
We generalize the classical Bernstein theorem concerning the constructive description of classes of functions uniformly continuous on the real line. The approximation of continuous bounded functions by entire functions of exponential type on unbounded closed proper subsets of the real line is studded.
\end{abstract}

\section{Introduction and Main Results}

For a closed unbounded set $E \subset \mathbf{R}$, denote by $B C(E)$ the class of (complexvalued) functions which are bounded and continuous on $E$. Let $E_{\sigma}$ be the class of entire functions of exponential type at most $\sigma>0$ and let

$$
A_{\sigma}(f, E):=\inf _{g \in E_{\sigma}}\|f-g\|_{C(E)} \quad(f \in B C(E)),
$$

where $\|\cdot\|_{C(E)}$ means the uniform norm over $E$.

The classical Bernstein direct and inverse theorems (see [22, p.p. 257, 340]) describe the relations between the smoothness of $f \in B C(\mathbf{R})$ and the rate of decrease of $A_{\sigma}(f, \mathbf{R})$ as $\sigma \rightarrow \infty$. In particular, from Bernstein's results it follows that for $f \in B C(\mathbf{R})$ and $0<\alpha<1$,

$$
A_{\sigma}(f, \mathbf{R})=O\left(\sigma^{-\alpha}\right) \quad \text { as } \sigma \rightarrow \infty
$$

if and only if

$$
\omega_{f, \mathbf{R}}(\delta)=O\left(\delta^{\alpha}\right) \quad \text { as } \delta \rightarrow+0,
$$

where

$$
\omega_{f, \mathbf{R}}(\delta):=\sup _{\substack{x_{1}, x_{2} \in \mathbf{R} \\\left|x_{1}-x_{2}\right| \leq \delta}}\left|f\left(x_{2}\right)-f\left(x_{1}\right)\right| \quad(\delta>0) .
$$

* The work was supported in part by NSF grant DMS-0554344 and DFG grant BL 272/101.

Date received: . Communicated by

AMS classification: $30 \mathrm{C} 10,30 \mathrm{E} 10$

Key words and phrases: Entire functions of exponential type, Approximation, Continuous function. 
The main objective of this paper is to extend Bernstein's results to the case where the function is considered on a proper subset of $\mathbf{R}$. We mostly focus on the case where the number of components of $E$ is infinite. Some aspects of this problem are considered in the recent papers by Shirokov [18], [19].

Let

$$
d(A, B):=\operatorname{dist}(A, B)=\inf _{z \in A, \zeta \in B}|z-\zeta| \quad(A, B \subset \mathbf{C}),
$$

and let $|B|$ denote the one-dimensional Lebesgue measure (length) of $B \subset \mathbf{C}$.

Unless otherwise stated, we denote by $C, C_{1}, C_{2}, \ldots$ positive constants that are either absolute or depend on $E$ only.

The set $E^{*}:=\mathbf{R} \backslash E$ consists of a finite or infinite number of disjoint open intervals $J_{j}=\left(a_{j}, b_{j}\right)$. In the reminder of this paper we assume that $E$ possesses the following two properties: for any $j$ under consideration,

$$
\begin{gathered}
\left|J_{j}\right| \leq C_{1}, \\
\sum_{k \neq j}\left(\frac{\left|J_{k}\right|}{d\left(J_{k}, J_{j}\right)}\right)^{2} \leq C_{2} .
\end{gathered}
$$

We use the following examples to illustrate the forthcoming results and constructions. The examples show that the number of "holes" $J_{j}$ can be infinite.

Example 1. Let

$$
d_{l-1}<c_{l}<d_{l}<c_{l+1} \quad(l=0, \pm 1, \pm 2, \ldots)
$$

be such that

$$
d_{l}-c_{l} \geq C_{3}, \quad c_{l+1}-d_{l} \leq C_{4}
$$

Then, the set

$$
E_{1}=\cup_{l=-\infty}^{\infty}\left[c_{l}, d_{l}\right]
$$

satisfies (1.3) and (1.4).

Example 2. A direct computation shows that the set $E_{2}=\mathbf{R} \backslash E_{2}^{*}$, where

$$
\begin{aligned}
E_{2}^{*}=\cup_{j=-\infty}^{\infty} \cup_{k=2}^{\infty} \quad & \left\{\left(2 j+2^{-k}\left(1-k^{-1}\right), 2 j+2^{-k}\right)\right. \\
& \left.\cup\left(2 j-2^{-k}, 2 j-2^{-k}\left(1-k^{-1}\right)\right)\right\}
\end{aligned}
$$

also satisfies (1.3) and (1.4).

In the case of polynomial approximation of continuous functions on a finite interval $[a, b] \subset \mathbf{R}$, the special role of the endpoints $a$ and $b$ is well-known. An elegant idea, suggested in [8], is to introduce a new modulus of continuity by 
using the distance between the points on $[a, b]$ that is not Euclidean. In the case of entire function approximation on $E$ the endpoints of $J_{j}$ also play a special role. We capture this effect by making use of a special distance between points of $E$ in the definition of the modulus of continuity of a function $f \subset B C(E)$. This distance is defined as follows. Let $\mathbf{H}:=\{z: \Im z>0\}$ be the upper half-plane. According to Levin [15] there exist vertical intervals $J_{j}^{\prime}=\left(u_{j}, u_{j}+i v_{j}\right], u_{j} \in$ $\mathbf{R}, v_{j}>0$ and a conformal mapping

$$
\phi: \mathbf{H} \rightarrow \mathbf{H}_{E}:=\mathbf{H} \backslash\left(\cup_{j} J_{j}^{\prime}\right)
$$

normalized by $\phi(\infty)=\infty, \phi(i)=i$ such that $\phi$ can be extended continuously to $\overline{\mathbf{H}}$ and it satisfies the boundary correspondence $\phi\left(J_{j}\right)=J_{j}^{\prime}$. For $x_{1}, x_{2} \in E$ such that $x_{1}<x_{2}$ set

$$
\rho_{E}\left(x_{1}, x_{2}\right)=\rho_{E}\left(x_{2}, x_{1}\right):=\operatorname{diam} \phi\left(\left[x_{1}, x_{2}\right]\right),
$$

where

$$
\operatorname{diam} B:=\sup _{z, \zeta \in B}|z-\zeta| \quad(B \subset \mathbf{C}) .
$$

In spite of its definition via the conformal mapping, the behavior of $\rho_{E}$ can be characterized in purely geometrical terms as follows. According to (1.4)

$$
d\left(J_{j}, E^{*} \backslash J_{j}\right) \geq C_{5}\left|J_{j}\right|, \quad C_{5}=C_{2}^{-1 / 2} .
$$

Let constant $C$ be fixed such that $0<C<\min \left(1, C_{5} / 2\right)$. For any component $J_{j}$ of $E^{*}$, denote by $\tilde{J}_{j}$ the open interval with the same center of the length $(1+C)\left|J_{j}\right|$. For $x_{1}, x_{2} \in E$ such that $x_{1}<x_{2}$ consider the function

$$
\begin{gathered}
\tau_{E}\left(x_{1}, x_{2}\right)=\tau_{E}\left(x_{2}, x_{1}\right)=\tau_{E, C}\left(x_{1}, x_{2}\right) \\
:= \begin{cases}\left(\frac{\left|J_{j}\right|}{d\left(\left[x_{1}, x_{2}\right], J_{j}\right)}\right)^{1 / 2}\left(x_{2}-x_{1}\right), & \text { if } x_{1}, x_{2} \in \tilde{J}_{j} \text { for some } j \text { and } \\
& x_{2}-x_{1}<d\left(\left[x_{1}, x_{2}\right], J_{j}\right), \\
\left|J_{j}\right|^{1 / 2}\left(x_{2}-x_{1}\right)^{1 / 2}, & \text { if } x_{1}, x_{2} \in \tilde{J}_{j} \text { for some } j \text { and } \\
& d\left(\left[x_{1}, x_{2}\right], J_{j}\right) \leq x_{2}-x_{1} \leq \frac{C}{2}\left|J_{j}\right|, \\
x_{2}-x_{1}, & \text { otherwise. }\end{cases}
\end{gathered}
$$

Theorem 1 For $x_{1}, x_{2} \in E$,

$$
\frac{1}{C_{6}} \tau_{E}\left(x_{1}, x_{2}\right) \leq \rho_{E}\left(x_{1}, x_{2}\right) \leq C_{6} \tau_{E}\left(x_{1}, x_{2}\right),
$$

where $C_{6}=C_{6}(E, C)>1$. 
Notice that according to Theorem 1

$$
\rho_{E}\left(x_{1}, x_{2}\right) \geq C_{7}\left|x_{2}-x_{1}\right| \quad\left(x_{1}, x_{2} \in E\right) .
$$

The main result of this paper is the following analogue of (1.1)-(1.2): for $f \in$ $B C(E)$ and $0<\alpha<1$,

$$
A_{\sigma}(f, E)=O\left(\sigma^{-\alpha}\right) \quad \text { as } \sigma \rightarrow \infty
$$

if and only if

$$
\omega_{f, E}^{*}(\delta)=O\left(\delta^{\alpha}\right) \quad \text { as } \delta \rightarrow+0,
$$

where

$$
\omega_{f, E}^{*}(\delta):=\sup _{\substack{x_{1}, x_{2} \in E \\ \rho_{E}\left(x_{1}, x_{2}\right) \leq \delta}}\left|f\left(x_{2}\right)-f\left(x_{1}\right)\right| \quad(\delta>0) .
$$

The statement (1.8)-(1.9) follows immediately from the direct Theorem 2 and the inverse Theorem 3 below.

Let $\omega(\delta), \delta>0$ be a function of modulus of continuity type, i.e., a positive nondecreasing function with $\omega(+0)=0$ such that

$$
\omega(t \delta) \leq 2 t \omega(\delta) \quad(\delta>0, t>1) .
$$

Denote by $B C_{\omega}^{*}(E)$ the class of functions $f \in B C(E)$ satisfying

$$
\omega_{f, E}^{*}(\delta) \leq \omega(\delta) \quad(\delta>0) .
$$

Theorem 2 For $f \in B C_{\omega}^{*}(E)$ and $\sigma \geq 1$,

$$
A_{\sigma}(f, E) \leq C_{8}\left(\frac{\|f\|_{C(E)}}{\sigma}+\omega\left(\frac{1}{\sigma}\right)\right) .
$$

Theorem 3 Let $f \in B C(E)$ and let

$$
A_{\sigma}(f, E) \leq \omega\left(\frac{1}{\sigma}\right) \quad(\sigma \geq 1) .
$$

Then for $x_{1}, x_{2} \in E$,

$$
\left|f\left(x_{2}\right)-f\left(x_{1}\right)\right| \leq C_{9} \Omega\left(\rho_{E}\left(x_{1}, x_{2}\right)\right),
$$

where

$$
\Omega(\delta):=\delta\left(\|f\|_{C(E)}+\int_{\delta}^{1} \frac{\omega(t)}{t^{2}} d t\right) \quad\left(0<\delta \leq \frac{1}{2}\right)
$$

and $\Omega(\delta):=\Omega(1 / 2)$ for $\delta>1 / 2$. 
Let us introduce the notation we will be using throughout the paper. We continue to use the convention that $C, C_{1}, \ldots$ denote positive constants, different in different sections and depending only on inessential quantities. For $a, b \geq 0$ we write $a \preceq b$ if $a \leq C b$. We also write $a \asymp b$ if $a \preceq b$ and $b \preceq a$ simultaneously.

Let for $z \in \mathbf{C}$ and $\delta>0$,

$$
\begin{gathered}
D(z, \delta):=\{\zeta:|\zeta-z|<\delta\}, \quad C(z, \delta):=\{\zeta:|\zeta-z|=\delta\}, \\
D(\delta):=D(0, \delta), \quad C(\delta):=C(0, \delta), \\
\mathbf{H}_{+}:=\mathbf{H}, \quad \mathbf{H}_{-}:=\mathbf{C} \backslash \overline{\mathbf{H}_{+}} \\
\mathbf{N}:=\{1,2, \ldots\}, \quad \mathbf{N}_{0}:=\{0,1, \ldots\} .
\end{gathered}
$$

The rest of the paper is organized as follows. Since a significant number of proofs in this paper depends on the techniques for estimation of the module of path families, Section 2 contains a brief summary of the appropriate results from geometric function theory. In Section 3, we compile certain facts about the Levin conformal mapping. In particular, the proof of Theorem 1 is given in this section. Sections 4-6 present preliminary results for the proof of Theorem 2 given in Section 7. Specifically, in Section 4, we construct auxiliary domains and study their conformal mappings onto a half-plane. Section 5 summarizes the relevant material on the continuous extension of functions from a closed subset of $\mathbf{R}$ into $\mathbf{C}$. Section 6 provides an exposition of some facts from the theory of entire functions. In Section 8, we prove Theorem 3.

\section{Auxiliary Results about Conformal Mappings}

In this section we discuss mostly known results which concern the distortion properties of some conformal mappings. The results are stated in the form convenient for further exposition.

As usual, a Jordan curve is a continuous image of a closed interval without intersections (except possibly endpoints). By a curve we understand a locally rectifiable Jordan curve without endpoints. We define a path to be the union of finitely many mutually disjoint curves. We use $\Gamma, \Gamma_{1}, \ldots$ to denote path families. We may use the same symbol for different families if it does not lead to confusion. For a path family $\Gamma$ denote by $m(\Gamma)$ its module, see [2], [14], and [11]. In the sequel we refer to the basic properties of the module, such as conformal invariance, comparison principle, composition laws, etc. (see monographs cited above for more details). As a rule, we will use these properties without further citations.

Special families of separating paths play a useful role. Let $G \subset \mathbf{C}$ be a domain. We say that a path $\gamma \in G$ separates sets $A \subset \bar{G}$ and $B \subset \bar{G}$ if $\gamma$ consists of 
a finite number of crosscuts of $G$ and any curve $J \subset G$ joining $A$ with $B$ has nonempty intersection with $\gamma$. We denote by $\Gamma(A ; B ; G)$ the set of all such paths. Sometimes, more sophisticated families of separating curves are used. Let $\tilde{G}$ be a compactification of $G$ by prime ends in the Carathéodory sense (see $[17])$. A point $z \in G$ can also be understood as a prime end $Z \in \tilde{G}$ defined by a chain of concentric circles converging to this point. We say that a crosscut $\gamma \subset G$ separates $Z_{1}, Z_{2}, \ldots \in \tilde{G}$ from $\mathcal{Z}_{1}, \mathcal{Z}_{2}, \ldots \in \tilde{G}$ in $G$ if $G \backslash \gamma$ consists of two connected components such that one of them is adjacent to $Z_{1}, Z_{2}, \ldots$ and the other to $\mathcal{Z}_{1}, \mathcal{Z}_{2}, \ldots$ (the adjacency means that in the domain and the subdomain a prime end can be defined by the same chain of crosscuts or concentric circles). We denote by $\Gamma\left(Z_{1}, Z_{2}, \ldots ; \mathcal{Z}_{1}, \mathcal{Z}_{2}, \ldots ; G\right)$ the set of all such crosscuts.

The examples below state some well-known facts concerning special path families.

Example 3. For $0<\alpha \leq 2 \pi$ and $0<r_{1}<r_{2}$, let

$$
\Gamma_{1}=\left\{\gamma_{r}=\left\{r e^{i \theta}: 0<\theta<\alpha \pi\right\}: r_{1}<r<r_{2}\right\}
$$

Then

$$
m\left(\Gamma_{1}\right)=\frac{1}{\alpha \pi} \log \frac{r_{2}}{r_{1}}
$$

(see [7, p. 8]).

Example 4. Let $z_{1}, z_{2}, z_{3} \in \mathbf{C}$ be distinct points satisfying $\left|z_{1}-z_{2}\right| \leq\left|z_{1}-z_{3}\right|$. Then, for the module of $\Gamma_{2}=\Gamma\left(z_{1}, z_{2} ; z_{3}, \infty ; \mathbf{C}\right)$ we have

$$
\frac{1}{2 \pi} \log \left|\frac{z_{1}-z_{3}}{z_{1}-z_{2}}\right| \leq m\left(\Gamma_{2}\right) \leq \frac{1}{2 \pi} \log \left|\frac{z_{1}-z_{3}}{z_{1}-z_{2}}\right|+2
$$

(see [7, pp. 98-99]).

Example 5. Let $z_{1} \in \mathbf{R}$ and $z_{2}, z_{3} \in \overline{\mathbf{H}}$ be distinct points and let $\Gamma_{3}=$ $\Gamma\left(z_{1}, z_{2} ; z_{3}, \infty ; \mathbf{H}\right)$. If $\left|z_{1}-z_{2}\right| \leq\left|z_{1}-z_{3}\right|$ then

$$
\frac{1}{\pi} \log \left|\frac{z_{1}-z_{3}}{z_{1}-z_{2}}\right| \leq m\left(\Gamma_{3}\right) \leq \frac{1}{\pi} \log \left|\frac{z_{1}-z_{3}}{z_{1}-z_{2}}\right|+2 .
$$

If $\left|z_{1}-z_{2}\right| \geq\left|z_{1}-z_{3}\right|$ then

$$
m\left(\Gamma_{3}\right) \leq 2
$$

(cf. [7, p. 35]).

Example 6. For $z \in \mathbf{H}$ and $\zeta \in \overline{\mathbf{H}}$ let $\Gamma_{4}=\Gamma(z ; \zeta, \infty ; \mathbf{H})$. Reasoning similar to that in the proof of (2.3) and (2.4) demonstrates that if $\Im z<|\zeta-\Re z|$ then

$$
\frac{1}{\pi} \log \frac{|\zeta-\Re z|}{\Im z} \leq m\left(\Gamma_{4}\right) \leq \frac{1}{\pi} \log \frac{|\zeta-\Re z|}{\Im z}+2,
$$


and if $\Re z=\zeta \in \mathbf{R}$, then

$$
m\left(\Gamma_{4}\right) \leq 2 .
$$

Example 7. Let $0<r<R$ and let curves $l_{r} \subset D(r) \cap \mathbf{H}$ and $l_{R} \subset \mathbf{H} \backslash D(R)$ be such that

$$
0 \in l_{r}, \infty \in l_{R}, \quad C(r) \cap \overline{l_{r}} \neq \emptyset, C(R) \cap \overline{l_{R}} \neq \emptyset .
$$

Denote by $\Gamma_{5}$ the family of all crosscuts of $G=\mathbf{H} \backslash\left(l_{r} \cup l_{R}\right)$ joining $l_{r}$ to $l_{R}$. Then

$$
m\left(\Gamma_{5}\right) \geq \frac{\pi}{2 \pi+\log \frac{R}{r}} .
$$

Indeed, let $z_{r} \in C(r) \cap \overline{l_{r}}$ and $z_{R} \in C(R) \cap \overline{l_{R}}$. According to (2.3), for the family $\Gamma_{6}$ of all crosscuts of $G$ which separate $l_{r}$ and $l_{R}$ we have

$$
m\left(\Gamma_{6}\right) \leq m\left(\Gamma\left(0, z_{r} ; z_{R}, \infty, \mathbf{H}\right)\right) \leq \frac{1}{\pi} \log \frac{R}{r}+2
$$

Since $m\left(\Gamma_{5}\right) m\left(\Gamma_{6}\right)=1$, we obtain $(2.7)$.

Example 8. Let $0<r<R$ and let $S_{ \pm}$be open sets consisting of disjoint intervals $\left(c_{j}^{ \pm}, d_{j}^{ \pm}\right)$such that

$$
\begin{gathered}
S_{+}=\cup_{j}\left(c_{j}^{+}, d_{j}^{+}\right) \subset(r, R), \quad S_{-}=\cup_{j}\left(c_{j}^{-}, d_{j}^{-}\right) \subset(-R,-r), \\
d\left(\left(c_{j}^{ \pm}, d_{j}^{ \pm}\right),\{0\} \cup S_{ \pm} \backslash\left(c_{j}^{ \pm}, d_{j}^{ \pm}\right)\right) \geq C_{1}\left(d_{j}^{ \pm}-c_{j}^{ \pm}\right), \\
\sum_{j}\left(\frac{d_{j}^{ \pm}-c_{j}^{ \pm}}{\min \left\{c_{j}^{ \pm}, d_{j}^{ \pm}\right\}}\right)^{2} \leq C_{2} .
\end{gathered}
$$

Denote by $\Gamma_{7}$ the family of all paths in

$$
Q=\{z \in \mathbf{H}: r<|z|<R\}
$$

which separate $(r, R) \backslash S_{+}$from $(-R,-r) \backslash S_{-}$.

Lemma 1 Under the above assumptions,

$$
m\left(\Gamma_{7}\right) \leq\left(\pi \log \frac{R}{r}+C_{3}\right)\left(\log \frac{R}{r}\right)^{-2},
$$

where $C_{3}=C_{3}\left(C_{1}, C_{2}\right)$.

Proof. We essentially follow the outline of the proof of [6, Lemma 4]. Let $U_{j}^{ \pm}$be the open interval with the center in $\left(c_{j}^{ \pm}+d_{j}^{ \pm}\right) / 2$ and the length $\left(1+C_{1} / 3\right)\left(d_{j}^{ \pm}-\right.$ $\left.c_{j}^{ \pm}\right)$. Let

$$
V_{j}^{+}=\left(e_{j}^{+}, f_{j}^{+}\right):=(r, R) \cap U_{j}^{+}, \quad V_{j}^{-}=\left(e_{j}^{-}, f_{j}^{-}\right):=(-R,-r) \cap U_{j}^{-},
$$


and let

$$
\begin{aligned}
& R_{j}^{+}:=\left\{z=r e^{i \theta}: e_{j}^{+} \leq r \leq f_{j}^{+}, 0 \leq \theta \leq C_{4} \log \frac{f_{j}^{+}}{e_{j}^{+}}\right\}, \\
& R_{j}^{-}:=\left\{z=r e^{i \theta}:-f_{j}^{-} \leq r \leq-e_{j}^{-}, \pi-C_{4} \log \frac{e_{j}^{-}}{f_{j}^{-}} \leq \theta \leq \pi\right\},
\end{aligned}
$$

where, by virtue of (2.8), $C_{4}$ can be chosen such that

$$
\log \frac{f_{j}^{+}}{e_{j}^{+}} \leq \frac{\pi}{2 C_{4}}, \quad \log \frac{e_{j}^{-}}{f_{j}^{-}} \leq \frac{\pi}{2 C_{4}} .
$$

Consider the metrics

$$
\begin{gathered}
\rho^{*}(z)= \begin{cases}|z|^{-1}, & \text { if } z \in Q, \\
0, & \text { elsewhere in } \mathbf{C},\end{cases} \\
\rho_{j}^{ \pm}(z)= \begin{cases}C_{5}\left(f_{j}^{ \pm}-e_{j}^{ \pm}\right)^{-1}, & \text { if } z \in R_{j}^{ \pm}, \\
0, & \text { elsewhere in } \mathbf{C},\end{cases}
\end{gathered}
$$

where $C_{5}$ is chosen such that for any $\gamma \in \Gamma_{7}$ with $\bar{\gamma} \cap\left[c_{j}^{ \pm}, d_{j}^{ \pm}\right] \neq \emptyset$ we have

$$
\int_{\gamma} \rho_{j}^{ \pm}(z)|d z| \geq 1
$$

According to our construction, for an arbitrary $\gamma \in \Gamma_{7}$ and

$$
\rho(z)=\max \left\{\rho^{*}(z), \sum_{ \pm} \sum_{j} \rho_{j}^{ \pm}(z)\left|\log \frac{f_{j}^{ \pm}}{e_{j}^{ \pm}}\right|\right\}
$$

we obtain

$$
\int_{\gamma} \rho(z)|d z| \geq \log \frac{R}{r}
$$

Since by $(2.9)$

$$
\begin{aligned}
& \int_{\mathbf{C}} \rho(z)^{2} d m(z) \\
\leq & \int_{\mathbf{C}} \rho^{*}(z)^{2} d m(z)+\sum_{ \pm} \sum_{j}\left(\int_{\mathbf{C}} \rho_{j}^{ \pm}(z)^{2} d m(z)\right)\left|\log \frac{f_{j}^{ \pm}}{e_{j}^{ \pm}}\right|^{2} \\
\leq & \pi \log \frac{R}{r}+C_{6},
\end{aligned}
$$


where $d m(z)$ stands for the 2 -dimensional Lebesgue measure (area) on $\mathbf{C}$, the definition of the module (see [14, p. 132]) as well as (2.11) and (2.12) yield (2.10) with $C_{3}=C_{6}$.

Next, we cite a result by Jenkins and Oikawa [12] concerning Ahlfors' fundamental inequalities.

Lemma 2 ([12, inequalities (1) and (3)]) For $0<r_{1}<r_{2}<\infty$, let

$$
Q=Q\left(r_{1}, r_{2}\right):=\left\{r e^{i \theta}: r_{1}<r<r_{2},-\theta_{1}(r)<\theta<\theta_{2}(r)\right\},
$$

where the functions $\theta_{j}, j=1,2$ have finite total variation $V_{j}$ on $\left[r_{1}, r_{2}\right]$ and satisfy

$$
0<\theta_{0} \leq \theta_{j}(r) \leq 2 \pi
$$

Then, for the module of $Q$, i.e., the module of the family $\Gamma=\Gamma(Q)$ of curves separating in $Q$ its boundary circular components, we have

$$
\int_{r_{1}}^{r_{2}} \frac{d r}{\left(\theta_{1}(r)+\theta_{2}(r)\right) r} \leq m(\Gamma) \leq \int_{r_{1}}^{r_{2}} \frac{d r}{\left(\theta_{1}(r)+\theta_{2}(r)\right) r}+\frac{\pi}{\theta_{0}^{2}}\left(V_{1}+V_{2}\right) .
$$

In the proof of Theorem 2 we use a statement whose analogue for the case of a domain including $\infty$ is due to Belyi (see [7, pp. 65-66]).

Let $G \subset \mathbf{C}$ be a simply connected domain such that $i \in G$ and $\infty \in L:=\partial G$. Denote by $\Phi: G \rightarrow \mathbf{H}$ a conformal mapping satisfying $\Phi(i)=i, \Phi(\infty)=\infty$ and let $\Psi:=\Phi^{-1}$. For $\xi \in \mathbf{D}^{*}:=\{\xi:|\xi|>1\}$ denote by $\Gamma_{8}(\xi)$ the family of all crosscuts of $\mathbf{D}^{*}$ which separate $\xi$ from $\bar{\xi}$. The module of $\Gamma_{8}(\xi)$ satisfies

$$
m\left(\Gamma_{8}(\xi)\right) \leq \frac{2}{\pi} \log \frac{4 d(\xi,\{1,-1\})}{|\xi|-1}
$$

(see [5, p. 113]). For $\tau \in \mathbf{H}, \Re \tau \neq 0$ denote by $\Gamma_{9}(\tau)$ the family of all crosscuts of $\mathbf{H}$ which separate $\tau$ from $-\bar{\tau}$. Conformal invariance of the module implies that

$$
m\left(\Gamma_{9}(\tau)\right)=m\left(\Gamma_{8}(\xi)\right), \quad \xi=\frac{\tau+i}{\tau-i} .
$$

Let $a=\Re \tau \neq 0$ and $0<b=\Im \tau \leq 1 / 2$. Elementary computation involving (2.13) and (2.14) shows that

$$
m\left(\Gamma_{9}(\tau)\right) \leq \frac{2}{\pi} \log \frac{8 \sqrt{2}(|a|+b)}{b}
$$


Lemma 3 Let $\tau_{1}$ and $\tau_{2}$ be such that $0<\Im \tau_{1}=\Im \tau_{2} \leq 1 / 2, \Re\left(\tau_{2}-\tau_{1}\right)=2 a \neq 0$ and let $\zeta_{j}=\Psi\left(\tau_{j}\right), j=1,2$. Then

$$
\frac{\left|\zeta_{1}-\zeta_{2}\right|}{d\left(\zeta_{1}, L\right)} \leq C\left(1+\frac{|a|}{b}\right)^{4}, \quad C=2^{14} .
$$

Proof. According to $(2.15)$ for $\Gamma_{10}=\Gamma\left(\tau_{1} ; \tau_{2} ; \mathbf{H}\right)$

$$
m\left(\Gamma_{10}\right) \leq \frac{2}{\pi} \log \frac{8 \sqrt{2}(|a|+b)}{b} .
$$

In order to prove (2.16) we can assume that

$$
\left|\zeta_{1}-\zeta_{2}\right|>d:=d\left(\zeta_{1}, L\right)
$$

By virtue of (2.1), for

$$
\Gamma_{11}=\left\{C\left(\zeta_{1}, r\right): d<r<\left|\zeta_{1}-\zeta_{2}\right|\right\}
$$

we obtain

$$
m\left(\Gamma_{11}\right)=\frac{1}{2 \pi} \log \frac{\left|\zeta_{1}-\zeta_{2}\right|}{d} .
$$

Since any $\gamma \in \Gamma_{11}$ includes a subarc which belongs to $\Psi\left(\Gamma_{10}\right)$, we have

$$
m\left(\Gamma_{11}\right) \leq m\left(\Psi\left(\Gamma_{10}\right)\right)=m\left(\Gamma_{10}\right)
$$

Comparing the above inequality with (2.17) and (2.18) we obtain (2.16).

Lemma 4 Let $\Im \tau_{1}=1 / \sigma, \sigma \geq 2, \tau_{2}=\tau_{1}+t, t \in \mathbf{R}, \zeta_{j}=\Psi\left(\tau_{j}\right), j=1,2$. Then for $z \in \mathbf{C} \backslash G$,

$$
\left|\frac{\zeta_{2}-z}{\zeta_{1}-z}\right| \leq C(1+\sigma|t|)^{4} \quad C=2^{14}+1 .
$$

Proof. Letting $a=t / 2$ and $b=1 / \sigma$ in Lemma 3 we have

$$
\left|\frac{\zeta_{2}-z}{\zeta_{1}-z}\right| \leq\left|\frac{\zeta_{2}-\zeta_{1}}{\zeta_{1}-z}\right|+1 \leq\left(2^{14}+1\right)(1+\sigma|t|)^{4} .
$$

The domains which appear in this paper do not have inner cusps on the boundary. That is, following [21] we say that $\mathbf{C} \backslash G=: K \in H$ if any points $z, \zeta \in K$ can be joined by a curve $\gamma(z, \zeta) \subset K$ such that

$$
|\gamma(z, \zeta)| \leq C_{7}|z-\zeta|, \quad C_{7}=C_{7}(K) \geq 1
$$


In the case where $\infty \in G$ and $K \in H$ the geometric properties of $G$ are studied in [3], [4]. In this paper we assume that $\infty \in L=\partial G$ and formulate some obvious modifications of results and constructions from these papers.

For the rest of this section we assume that $K \in H$ and the constants in the inequalities depend only on $K$. Let $\tilde{G}$ be the compactification of $G$ by prime ends (see [17]) and let $\tilde{L}:=\tilde{G} \backslash G$. If $L$ is a Jordan curve, then $\tilde{L}=L$. Since $K \in H$ all $Z \in \tilde{L}$ are of first kind, i.e., they have singleton impressions $|Z|=z \in L$. For $Z \in \tilde{L}$ and $r>0$ denote by $\gamma_{Z}(r)=\gamma_{Z}(r, G) \subset G \cap C(z, r)$ a crosscut of $G$ which separates $Z$ from $\infty$. For our purposes it is sufficient to assume that for any $Z \in \tilde{L}$ and $r>0$ the crosscut $\gamma_{Z}(r)$ is defined uniquely. We use the same symbol $\Phi$ to denote the homeomorphism between $\tilde{G}$ and $\overline{\mathbf{H}}$ which coincides in $G$ with the mapping $\Phi$ and let $\Psi=\Phi^{-1}$.

First, note that for $Z \in \tilde{L}$ and $r>0$,

$$
\sup _{\zeta \in \gamma_{Z}(r)}|\Phi(\zeta)-\Phi(Z)| \asymp \inf _{\zeta \in \gamma_{Z}(r)}|\Phi(\zeta)-\Phi(Z)|
$$

(cf. [4, Lemma 2]).

If $0<r<R$ then $\gamma_{Z}(r)$ and $\gamma_{Z}(R)$ are the sides of some quadrilateral $Q_{Z}(r, R)=Q_{Z}(r, R, G) \subset G$ whose other two sides are parts of the boundary $L$. Let $m_{Z}(r, R)=m_{Z}(r, R, G)$ be the module of this quadrilateral, i.e., the module of the family of curves that separate the sides $\gamma_{Z}(r)$ and $\gamma_{Z}(R)$ in $Q_{Z}(r, R)$.

Lemma 5 ([4, Theorem 2]) Let $Z \in \tilde{L}$ and $0<r_{1}<r_{2}<r_{3}$. Then

$$
\begin{gathered}
0 \leq m_{Z}\left(r_{1}, r_{3}\right)-\left(m_{Z}\left(r_{1}, r_{2}\right)+m_{Z}\left(r_{2}, r_{3}\right)\right) \preceq 1, \\
\frac{1}{2 \pi} \log \frac{r_{2}}{r_{1}} \leq m_{Z}\left(r_{1}, r_{2}\right) \preceq \log \frac{r_{2}}{r_{1}}+1 .
\end{gathered}
$$

Moreover, for any $\zeta_{j} \in \gamma_{Z}\left(r_{j}\right), j=1,2$,

$$
m_{Z}\left(r_{1}, r_{2}\right) \leq m\left(\Gamma\left(Z, \zeta_{1} ; \zeta_{2}, \infty ; G\right)\right) \leq m_{Z}\left(r_{1}, r_{2}\right)+C_{8}
$$

Lemma 6 ([3, Lemmas 2 and 3]) Let $Z \in \tilde{L}, \zeta_{1}, \zeta_{2} \in G, w=\Phi(Z), \tau_{k}=$ $\Phi\left(\zeta_{k}\right), k=1,2$ be such that $\Re w=\Re \tau_{1}=\Re \tau_{2}, \Im \tau_{1}<\Im \tau_{2}$. Then

$$
\begin{gathered}
\left|z-\zeta_{1}\right| \preceq\left|z-\zeta_{2}\right|, \\
d\left(\zeta_{k}, L\right) \asymp\left|\zeta_{k}-z\right|, \\
\left|\frac{w-\tau_{1}}{w-\tau_{2}}\right|^{2} \preceq\left|\frac{z-\zeta_{1}}{z-\zeta_{2}}\right| \preceq\left|\frac{w-\tau_{1}}{w-\tau_{2}}\right|^{C} .
\end{gathered}
$$


The analysis of the proof of the left-hand side of (2.22), given in [3], shows that in the case $|Z|=z \in \mathbf{R}$ and $G \subset \mathbf{H}$, it can be replaced by a sharper one

$$
\left|\frac{w-\tau_{1}}{w-\tau_{2}}\right| \preceq\left|\frac{z-\zeta_{1}}{z-\zeta_{2}}\right| \text {. }
$$

For $Z \in \tilde{L}, \zeta \in G$, and $\delta>0$ set

$$
z_{\delta}:=\Psi(\Phi(Z)+i \delta), \quad \zeta_{\delta}:=\Psi(\Phi(\zeta)+i \delta) .
$$

Lemma 7 ([3, Lemma 4]) Under the above notation if $|\Phi(\zeta)-\Phi(Z)| \leq C_{9} \delta$ then

$$
\frac{1}{C_{10}}\left|z-z_{\delta}\right| \leq\left|\zeta-\zeta_{\delta}\right| \leq C_{10}\left|z-z_{\delta}\right|
$$

where $C_{10}=C_{10}\left(G, C_{9}\right) \geq 1$.

For $Z \in \tilde{L}, \mathcal{Z} \in \tilde{G}$ and $z=|Z|, \zeta=|\mathcal{Z}|$ we denote by $r_{Z}(\mathcal{Z})$ the supremum of those $r>0$ for which the $\operatorname{arc} \gamma_{Z}(r)$ separates $Z$ from $\mathcal{Z}$. By the definition of the class $H$ we have

$$
r_{Z}(\mathcal{Z}) \geq C_{11}|z-\zeta|
$$

Moreover, if $\zeta \in G$ satisfies $\Re \Phi(\zeta)=\Re \Phi(Z)$, then

$$
r_{Z}(\zeta) \leq C_{12}|z-\zeta|
$$

(cf. $[4$, p. 61]).

Lemma 8 Let $x \in \mathbf{R}, \delta>0, z \in \overline{\mathbf{H}} \cap C(x, \delta)$, and let $W=\Psi(x), w=|W|, T=$ $\Psi(z), t=|T|, w_{\delta}=\Psi(x+i \delta)$. Then

$$
|w-t| \preceq\left|w-w_{\delta}\right| .
$$

Proof. If $|w-t| \geq \frac{C_{12}}{C_{11}}\left|w-w_{\delta}\right|$ we consider

$$
\begin{gathered}
\Gamma_{1}=\Gamma(x, z ; x+i \delta, \infty ; \mathbf{H}), \\
\Gamma_{2}=\left\{C(w, r): C_{12}\left|w-w_{\delta}\right|<r<C_{11}|w-t|\right\} .
\end{gathered}
$$

According to (2.1), (2.4), (2.24), and (2.25)

$$
2 \geq m\left(\Gamma_{1}\right)=m\left(\Psi\left(\Gamma_{1}\right)\right) \geq m\left(\Gamma_{2}\right)=\frac{1}{2 \pi} \log \frac{C_{11}|w-t|}{C_{12}\left|w-w_{\delta}\right|},
$$

which implies (2.26). 


\section{The Levin Conformal Mapping}

In this section we discuss metric properties of the Levin conformal mapping $\phi$ introduced in Section 1. We extend $\phi$ continuously to $\mathbf{R}$. This extension can be understood in two different ways: either (i) for $x \in \mathbf{R}$ the value of $\phi(x)$ is a point from $\partial \mathbf{H}_{E}$ or (ii) values of $\phi$ on $\mathbf{R}$ are prime ends in $\tilde{\mathbf{H}}_{E} \backslash \mathbf{H}_{E}$. Below we denote either extension by the same letter $\Phi$.

Since by (1.3) and (1.5) $E$ is relatively dense with respect to the linear Lebesgue measure, i.e., there exist $C_{1}$ and $C_{2}$ such that

$$
\left|\left[x, x+C_{1}\right] \cap E\right| \geq C_{2} \quad(x \in \mathbf{R}),
$$

according to [16, Theorem 3.9] for any component $J_{j}=\left(a_{j}, b_{j}\right)$ of $E^{*}$ and its image $J_{j}^{\prime}:=\phi\left(J_{j}\right)$ we have

$$
\left|J_{j}^{\prime}\right| \preceq 1
$$

as well as

$$
|\phi(z)|+1 \asymp|z|+1 \quad(z \in \mathbf{H}) .
$$

The set $E$ consists of disjoint components which are either points or closed intervals. Denote by $T_{j}=\left[c_{j}, a_{j}\right]$ and $R_{j}=\left[b_{j}, d_{j}\right]$ the components of $E$ that adjoin $J_{j}$ and let $T_{j}^{\prime}:=\phi\left(T_{j}\right), R_{j}^{\prime}:=\phi\left(R_{j}\right)$.

Lemma 9 The following holds:

$$
\begin{aligned}
& \left|J_{j}^{\prime}\right| \preceq\left|T_{j}^{\prime}\right|, \\
& \left|J_{j}^{\prime}\right| \preceq\left|R_{j}^{\prime}\right| .
\end{aligned}
$$

Proof. In order to prove (3.2) we can assume that $\left|J_{j}^{\prime}\right|>\left|T_{j}^{\prime}\right|$. Consider families of crosscuts

$$
\Gamma_{1}=\Gamma\left(c_{j}, a_{j} ; b_{j}, \infty ; \mathbf{H}\right), \quad \Gamma_{2}=\left\{C\left(u_{j}, r\right) \cap \mathbf{H}:\left|T_{j}^{\prime}\right|<r<\left|J_{j}^{\prime}\right|\right\} .
$$

By virtue of (1.5), (2.1), and (2.3)

$$
\begin{gathered}
\frac{1}{\pi} \log \frac{\left|J_{j}^{\prime}\right|}{\left|T_{j}^{\prime}\right|}=m\left(\Gamma_{2}\right) \leq m\left(\phi\left(\Gamma_{1}\right)\right) \\
=m\left(\Gamma_{1}\right) \leq \frac{1}{\pi} \log \frac{\left|T_{j}\right|+\left|J_{j}\right|}{\left|T_{j}\right|}+2 \preceq 1,
\end{gathered}
$$

which establishes the formula (3.2).

Similar argument applies to the proof of inequality (3.3). 
Lemma 9 implies that $\mathbf{C} \backslash \mathbf{H}_{E} \in H$, i.e., $\phi$ and its inverse mapping $\psi:=\phi^{-1}$ satisfy the properties mentioned in Section 2. In particular, let for $x \in E$ and $\delta>0$,

$$
E_{\delta}:=\{z \in \overline{\mathbf{H}}: \Im \phi(z)=\delta\}, \quad \rho_{\delta}(x):=d\left(x, E_{\delta}\right) .
$$

Denote by $x_{\delta}^{*} \in E_{\delta}$ any point satisfying $\Re x_{\delta}^{*}=\Re x$. We claim that

$$
\left|x-x_{\delta}^{*}\right| \preceq \rho_{\delta}(x) .
$$

Indeed, let $z \in E_{\delta}$ be such that $|x-z|=\rho_{\delta}(x)$. If $\left|x-x_{\delta}^{*}\right|>\rho_{\delta}(x)$, consider families of curves

$$
\left.\Gamma_{1}=\Gamma\left(x, z ; x_{\delta}^{*}, \infty ; \mathbf{H}\right), \quad \Gamma_{2}=\left\{C(x, r) \cap \mathbf{H}: \rho_{\delta}(x)<r<\left|x-x_{\delta}^{*}\right|\right\}\right\} .
$$

According to (2.1), (2.21), and Lemma 5

$$
\frac{1}{\pi} \log \frac{\left|x-x_{\delta}^{*}\right|}{\rho_{\delta}(x)}=m\left(\Gamma_{2}\right) \leq m\left(\Gamma_{1}\right)=m\left(\phi\left(\Gamma_{1}\right)\right) \preceq 1,
$$

which yields (3.4).

Comparing (3.4) and (2.21)-(2.23) for $0<\delta<\Delta$ we obtain

$$
\left(\frac{\delta}{\Delta}\right)^{1 / C} \preceq \frac{\rho_{\delta}(x)}{\rho_{\Delta}(x)} \asymp\left|\frac{x-x_{\delta}^{*}}{x-x_{\Delta}^{*}}\right| \preceq \frac{\delta}{\Delta},
$$

where $C$ is the constant from $(2.22)$.

Lemma 10 Let $x_{1}, x_{2} \in E, x_{1}<x_{2}, W_{k}=\phi\left(x_{k}\right) \in \tilde{\mathbf{H}}_{E} \backslash \mathbf{H}_{E},\left|W_{k}\right|=w_{k} \in$ $\mathbf{R}, k=1,2$. Then

$$
\operatorname{diam} \phi\left(\left[x_{1}, x_{2}\right]\right) \asymp\left|w_{k}-\phi\left(x_{k}+i\left(x_{2}-x_{1}\right)\right)\right| \quad(k=1,2) .
$$

Proof. We only consider the case $k=1$. The proof for the other case is similar. Let $\delta=x_{2}-x_{1}, \tau_{1}=\phi\left(x_{1}+i \delta\right)$. Consider the curve

$$
J=\left[x_{1}, x_{1}+i \delta\right] \cup\left\{z=\delta e^{i \theta}: 0 \leq \theta \leq \frac{\pi}{2}\right\} .
$$

By virtue of (2.20) and Lemma 8

$$
\operatorname{diam} \phi(J) \preceq\left|w_{1}-\tau_{1}\right|,
$$

which implies that

$$
B:=\operatorname{diam} \phi\left(\left[x_{1}, x_{2}\right]\right) \preceq\left|w_{1}-\tau_{1}\right| .
$$


To prove the opposite inequality, according to (2.24) we can assume that $B<$ $r_{W_{1}}\left(\tau_{1}\right)$. Further, consider

$$
\Gamma=\Gamma\left(W_{1}, W_{2} ; \tau_{1}, \infty ; \mathbf{H}_{E}\right)
$$

Lemma 5 and (2.4) yield

$$
\begin{aligned}
\frac{1}{2 \pi} \log \frac{r_{W_{1}}\left(\tau_{1}\right)}{B} & \leq m_{W_{1}}\left(B, r_{W_{1}}\left(\tau_{1}\right) ; \mathbf{H}_{E}\right) \\
& \leq m(\Gamma)=m(\psi(\Gamma)) \leq 2
\end{aligned}
$$

which, together with (2.24), implies that

$$
B \succeq r_{W_{1}}\left(\tau_{1}\right) \succeq\left|w_{1}-\tau_{1}\right|
$$

Comparing (3.7) and (3.8) we obtain (3.6).

Lemma 11 The following holds:

$$
\left|J_{j}^{\prime}\right| \asymp\left|J_{j}\right|
$$

Proof. For sufficiently large $R \in E$ consider

$$
\Gamma_{1}=\Gamma\left(a_{j}, b_{j} ; R, \infty ; \mathbf{H}\right), \quad \Gamma_{2}=\left\{C\left(u_{j}, r\right) \cap \mathbf{H}:\left|J_{j}^{\prime}\right|<r<\zeta_{R}-u_{j}\right\},
$$

where $\mathcal{Z}_{R}:=\phi(R)$ and $\zeta_{R}:=\left|\mathcal{Z}_{R}\right|$.

By (2.1) and (2.3)

$$
\begin{aligned}
\frac{1}{\pi} \log \frac{\zeta_{R}-u_{j}}{\left|J_{j}^{\prime}\right|} & =m\left(\Gamma_{2}\right) \leq m\left(\phi\left(\Gamma_{1}\right)\right) \\
& =m\left(\Gamma_{1}\right) \leq \frac{1}{\pi} \log \frac{R-a_{j}}{\left|J_{j}\right|}+2
\end{aligned}
$$

which implies that

$$
\frac{\left|J_{j}\right|}{\left|J_{j}^{\prime}\right|} \preceq \frac{R-a_{j}}{\zeta_{R}-u_{j}} .
$$

Letting $R \rightarrow \infty$ and using (3.1) we have

$$
\left|J_{j}^{\prime}\right| \succeq\left|J_{j}\right| .
$$


In order to prove the opposite inequality, for sufficiently large $R$ consider the family $\Gamma_{3}$ of all crosscuts of $\mathbf{H} \backslash\left(J_{j}^{\prime} \cup\left\{u_{j}+i v: v \geq R\right\}\right)$ joining $J_{j}^{\prime}$ with $\left\{u_{j}+i v: v \geq R\right\}$. Let $\Gamma_{4}=\left\{\gamma \cap \mathbf{H}_{E}: \gamma \in \Gamma_{3}\right\}$. According to (2.7)

$$
m\left(\Gamma_{4}\right) \geq m\left(\Gamma_{3}\right) \geq \pi\left(\log \frac{C_{3} R}{\left|J_{j}^{\prime}\right|}\right)^{-1} .
$$

Moreover, by virtue of (3.1) there is a constant $C_{4}$ such that for sufficiently large $R$

$$
\left|\psi\left(u_{j}+i v\right)-\frac{b_{j}-a_{j}}{2}\right| \geq C_{4} R \quad(v \geq R) .
$$

Let $\Gamma_{5}$ be the family of all paths in

$$
Q=\left\{z \in \mathbf{H}: \frac{\left|J_{j}\right|}{2}<\left|z-\frac{b_{j}+a_{j}}{2}\right|<C_{4} R\right\}
$$

which separate $(r, R) \backslash E^{*}$ from $(-R,-r) \backslash E^{*}$. By (1.4) and Lemma 1

$$
m\left(\Gamma_{4}\right)=m\left(\psi\left(\Gamma_{4}\right)\right) \leq m\left(\Gamma_{5}\right) \leq \pi\left(\log \frac{C_{5} R}{\left|J_{j}\right|}\right)\left(\log \frac{2 C_{4} R}{\left|J_{j}\right|}\right)^{-2}
$$

Comparing (3.11) and (3.12) and letting $R \rightarrow \infty$ we obtain

$$
\left|J_{j}^{\prime}\right| \preceq\left|J_{j}\right|,
$$

which gives (3.9) when combined with (3.10).

For $x \in E$ and $\delta>0$ let $W:=\phi(x), w:=|W|, w_{\delta}:=\phi(x+i \delta)$. The next three lemmas state estimates for the quantity $\left|w-w_{\delta}\right|$.

Lemma 12 The following holds:

$$
\left|w-w_{\delta}\right| \succeq \delta
$$

Proof. For sufficiently large $R \in E$ consider

$$
\begin{gathered}
\Gamma_{1}=\Gamma(x, x+i \delta ; R, \infty, \mathbf{H}), \\
\Gamma_{2}=\left\{C(w, r) \cap \mathbf{H}: C_{12}\left|w-w_{\delta}\right|<r<C_{11}\left|w-\zeta_{R}\right|\right\},
\end{gathered}
$$

where $C_{11}$ and $C_{12}$ are the constants from (2.24) and (2.25); and $\zeta_{R}$ is the impression of the prime end $\phi(R)$. According to (2.1) and (2.3)

$$
\begin{aligned}
& 2+\frac{1}{\pi} \log \frac{R-x}{\delta} \geq m\left(\Gamma_{1}\right)=m\left(\phi\left(\Gamma_{1}\right)\right) \\
\geq & m\left(\Gamma_{2}\right) \geq \frac{1}{\pi} \log \frac{C_{11}\left|\zeta_{R}-w\right|}{C_{12}\left|w_{\delta}-w\right|},
\end{aligned}
$$


from which, after taking into account (3.1) and letting $R \rightarrow \infty$, we obtain (3.14).

Let $\tilde{J}_{j}$ be as in Section 1, i.e., it is the open interval with the same center as $J_{j}$ and the length $(1+C)\left|J_{j}\right|$ where $C$ is sufficiently small.

Lemma 13 For $x \in \mathbf{R} \backslash \cup_{j} \tilde{J}_{j}$,

$$
\left|w-w_{\delta}\right| \preceq \delta .
$$

Proof. Essentially, we have to mimic the proof of (3.13). Hence, we only sketch it. For sufficiently large $R$ let

$$
\begin{gathered}
S=\{\tau=\phi(x+i t): 0 \leq t \leq \delta\}, \\
T=T_{R}=\{\tau=\phi(x+i t): t \geq R\} .
\end{gathered}
$$

Consider the family $\Gamma_{1}$ of curves joining $S$ and $T$ in $\mathbf{H} \backslash(S \cup T)$. Let

$$
\Gamma_{2}=\left\{\gamma \cap \mathbf{H}_{E}: \gamma \in \Gamma_{1}\right\} .
$$

According to (2.7), (2.20), and (3.1)

$$
m\left(\Gamma_{2}\right) \geq m\left(\Gamma_{1}\right) \geq \pi\left(\log \frac{C_{6} R}{\left|w-w_{\delta}\right|}\right)^{-1} .
$$

Furthermore, by virtue of (1.4) and Lemma 1 for the module of the family $\Gamma_{3}$ of all paths in

$$
Q=\{z \in \mathbf{H}: \delta<|z-x|<R\}
$$

which separate $(\delta, R) \backslash E^{*}$ from $(-R,-\delta) \backslash E^{*}$ we have

$$
m\left(\Gamma_{3}\right) \leq \pi\left(\log \frac{C_{7} R}{\delta}\right)\left(\log \frac{R}{\delta}\right)^{-2} .
$$

Since

$$
m\left(\Gamma_{2}\right)=m\left(\psi\left(\Gamma_{2}\right)\right) \leq m\left(\Gamma_{3}\right),
$$

comparing (3.16), (3.17) and letting $R \rightarrow \infty$ we obtain (3.15).

Lemma 14 Let $x \in \tilde{J}_{j}$ for some $j$.

(i) If $\delta \leq d\left(x, J_{j}\right)$, then

$$
\left|w-w_{\delta}\right| \asymp\left(\frac{\left|J_{j}\right|}{d\left(x, J_{j}\right)}\right)^{1 / 2} \delta .
$$


(ii) If $d\left(x, J_{j}\right)<\delta \leq\left|J_{j}\right|$, then

$$
\left|w-w_{\delta}\right| \asymp\left|J_{j}\right|^{1 / 2} \delta^{1 / 2} .
$$

(iii) If $\delta \geq\left|J_{j}\right|$, then

$$
\left|w-w_{\delta}\right| \asymp \delta .
$$

Proof. Without loss of generality we assume that $x \geq b_{j}$, i.e., $x \in R_{j}=\left[b_{j}, d_{j}\right]$. First, we provide some auxiliary estimates. According to the definition of $\tilde{J}_{j}$

$$
x-b_{j} \leq C\left|J_{j}\right|<\frac{1}{2}\left|R_{j}\right| .
$$

We claim that

$$
w-u_{j} \preceq v_{j}=\left|J_{j}^{\prime}\right| .
$$

Indeed, by (2.3), for the module of the family

$$
\Gamma_{1}=\Gamma\left(a_{j}, b_{j} ; x, \infty ; \mathbf{H}\right)
$$

we have

$$
m\left(\Gamma_{1}\right) \leq \frac{1}{\pi} \log \frac{x-a_{j}}{b_{j}-a_{j}}+2 \preceq 1 .
$$

on the other hand, if $w-u_{j}>v_{j}$, by virtue of (2.1)

$$
m\left(\Gamma_{1}\right)=m\left(\phi\left(\Gamma_{1}\right)\right) \geq m\left(\Gamma_{2}\right)=\frac{1}{\pi} \log \frac{w-u_{j}}{v_{j}},
$$

where

$$
\Gamma_{2}=\left\{C\left(u_{j}, r\right) \cap \mathbf{H}: v_{j}<r<w-u_{j}\right\} .
$$

Comparing (3.23) and (3.24) we obtain (3.22).

Furthermore,

$$
w-u_{j} \asymp\left(x-b_{j}\right)^{1 / 2}\left|J_{j}\right|^{1 / 2} .
$$

Indeed, by (2.3) and (3.21), for the module of the family

$$
\Gamma_{3}=\Gamma\left(x, b_{j} ; a_{j}, \infty ; \mathbf{H}\right)
$$

we have

$$
\frac{1}{\pi} \log \frac{\left|J_{j}\right|}{x-b_{j}} \leq m\left(\Gamma_{3}\right) \leq \frac{1}{\pi} \log \frac{\left|J_{j}\right|}{x-b_{j}}+C_{8} .
$$

On the other hand, making use of Lemmas 2 and 5 , we have

$$
\frac{2}{\pi} \log \frac{\left|J_{j}^{\prime}\right|}{w-u_{j}}-C_{9} \leq m\left(\phi\left(\Gamma_{3}\right)\right) \leq \frac{2}{\pi} \log \frac{\left|J_{j}^{\prime}\right|}{w-u_{j}}+C_{9},
$$


which, together with (3.9), (3.22), and (3.26), implies (3.25).

We are now in a position to prove (3.18)-(3.20). We compare the modules of pares of conformally invariant families of curves in $\mathbf{H}$ and $\mathbf{H}_{E}$. Since the reasoning mimics the proof of (3.25) we only indicate the appropriate choice of the families of crosscuts leaving the details to the reader.

(i) In this case, by (2.19) and Lemma $6,\left|w-w_{\delta}\right| \preceq w-u_{j}$ and for the family

$$
\Gamma_{4}=\Gamma\left(x, x+i \delta ; b_{j}, \infty ; \mathbf{H}\right)
$$

according to (2.3) and (2.4) we have

$$
\frac{1}{\pi} \log \frac{x-b_{j}}{\delta} \leq m\left(\Gamma_{4}\right) \leq \frac{1}{\pi} \log \frac{x-b_{j}}{\delta}+2,
$$

as well as

$$
\frac{1}{\pi} \log \frac{w-u_{j}}{\left|w-w_{\delta}\right|}-C_{10} \leq m\left(\phi\left(\Gamma_{4}\right)\right) \leq \frac{1}{\pi} \log \frac{w-u_{j}}{\left|w-w_{\delta}\right|}+C_{10} .
$$

Therefore,

$$
\left|w-w_{\delta}\right| \asymp \frac{w-u_{j}}{x-b_{j}} \delta,
$$

which, when combined with (3.25), gives (3.18).

(ii) In this case, by (2.19) and Lemma 6, w- $u_{j} \preceq\left|w-w_{\delta}\right| \preceq\left|J_{j}^{\prime}\right|$ and by virtue of (2.3) for the family

$$
\Gamma_{5}=\Gamma\left(x, x+i \delta ; a_{j}, \infty ; \mathbf{H}\right)
$$

we obtain

$$
\frac{1}{\pi} \log \frac{\left|J_{j}\right|}{\delta} \leq m\left(\Gamma_{5}\right) \leq \frac{1}{\pi} \log \frac{\left|J_{j}\right|}{\delta}+2 .
$$

Since, by Lemmas 2 and 5 ,

$$
\frac{2}{\pi} \log \frac{\left|J_{j}^{\prime}\right|}{\left|w-w_{\delta}\right|}-C_{11} \leq m\left(\phi\left(\Gamma_{5}\right)\right) \leq \frac{2}{\pi} \log \frac{\left|J_{j}^{\prime}\right|}{\left|w-w_{\delta}\right|}+C_{11},
$$

the two above double inequalities, together with (3.9), imply (3.19).

(iii) The part $\left|w-w_{\delta}\right| \succeq \delta$ follows from (3.14). In order to prove the opposite inequality $\left|w-w_{\delta}\right| \preceq \delta$ we have to repeat the proof of (3.13) word for word.

Proof of Theorem 1. According to Lemma 10

$$
\rho_{E}\left(x_{1}, x_{2}\right) \asymp\left|w_{1}-\tau_{1}\right|,
$$

where $w_{1}=\left|W_{1}\right|, W_{1}=\phi\left(x_{1}\right)$, and $\tau_{1}=\phi\left(x_{1}+i\left|x_{2}-x_{1}\right|\right)$. Therefore, Lemmas 12-14 imply (1.6). 


\section{Auxiliary Domains and Their Conformal Mappings}

In this section, starting with $E$ and $E^{*}=\cup_{j} J_{j}=\cup_{j}\left(a_{j}, b_{j}\right)$, we construct two auxiliary domains $G_{ \pm} \supset \mathbf{H}_{ \pm}$and study their conformal mappings onto $\mathbf{H}_{ \pm}$.

Consider the curves

$$
\begin{aligned}
S_{j}^{+}:= & {\left[a_{j}-2 i t_{j}, b_{j}-2 i t_{j}\right] \cup\left\{z:\left|z-\left(a_{j}-i t_{j}\right)\right|=t_{j}, \Re z \leq a_{j}\right\} } \\
& \cup\left\{z:\left|z-\left(b_{j}-i t_{j}\right)\right|=t_{j}, \Re z \geq b_{j}\right\},
\end{aligned}
$$

where $t_{j}:=C_{5}\left|J_{j}\right| / 3$ and $C_{5}$ is the constant from (1.5). Denote by $G_{+} \supset \mathbf{H}_{+}$ the Jordan domain bounded by

$$
L^{+}=\partial G_{+}=E \cup\left(\cup_{j} S_{j}^{+}\right)
$$

and let

$$
G_{-}:=\left\{z: \bar{z} \in G_{+}\right\}, \quad L^{-}:=\partial G_{-}, \quad S_{j}^{-}:=\left\{z: \bar{z} \in S_{j}^{+}\right\} .
$$

Denote by $\phi_{ \pm}: G_{ \pm} \rightarrow \mathbf{H}_{ \pm}$the conformal mapping normalized by

$$
\phi_{ \pm}(\infty)=\infty, \quad \phi_{ \pm}( \pm i)= \pm i
$$

and let $\psi_{ \pm}:=\phi_{ \pm}^{-1}$ be the inverse mapping. By the symmetry

$$
\phi_{+}(x)=\phi_{-}(x) \quad(x \in E) \text {. }
$$

Since, by (1.5) $\mathbf{C} \backslash G_{ \pm} \in H$, the conformal mappings $\phi_{ \pm}$and $\psi_{ \pm}$possess appropriate properties stated in Section 2.

Lemma 15 For $z \in G_{ \pm}$,

$$
\left|\phi_{ \pm}(z)\right|+1 \asymp|z|+1 \text {. }
$$

Proof. Let $w=w^{ \pm}=\phi_{ \pm}(z)$. Without loss of generality we can assume that $z \in$ $\mathbf{H}_{ \pm},|z|>2 d(i, E)$, and $|w|>1$. According to $(2.5)$, for $\Gamma_{1}=\Gamma\left( \pm i ; z, \infty ; G_{ \pm}\right)$ and $\Gamma_{2}=\Gamma\left( \pm i ; z, \infty ; \mathbf{H}_{ \pm}\right)$we have

$$
\frac{1}{\pi} \log |w| \leq m\left(\phi_{ \pm}\left(\Gamma_{1}\right)\right) \leq \frac{1}{\pi} \log |w|+2,
$$

as well as

$$
m\left(\Gamma_{1}\right) \leq m\left(\Gamma_{2}\right) \leq \frac{1}{\pi} \log |z|+2
$$


Comparing (4.3) and the left-hand side of (4.2) we obtain

$$
|w| \preceq|z| \text {. }
$$

Furthermore, let $x \in E$ be such that $|x \mp i|=d(i, E)=: d$ and let

$$
\Gamma_{3}=\left\{\gamma_{x}\left(r, G_{ \pm}\right): d<r<|z|-d\right\}
$$

Notice that

$$
\begin{aligned}
m\left(\Gamma_{1}\right) & \geq m\left(\Gamma_{3}\right)=\int_{d}^{|z|-d} \frac{d r}{\left|\gamma_{x}\left(r, G_{ \pm}\right)\right|} \\
& \geq \int_{d}^{|z| / 2} \frac{d r}{\pi r}-\frac{1}{\pi^{2}} \int_{d}^{|z|} \frac{\left|\gamma_{x}\left(r, G_{ \pm}\right)\right|-\pi r}{r^{2}} d r .
\end{aligned}
$$

Since, by our assumption (1.4)

$$
\int_{d}^{\infty} \frac{\left|\gamma_{x}\left(r, G_{ \pm}\right)\right|-\pi r}{r^{2}} d r \preceq 1,
$$

we have

$$
m\left(\Gamma_{1}\right) \geq \log |z|-C_{1},
$$

which, together with the right-hand side of (4.2), implies that

$$
|w| \succeq|z|
$$

Comparing the above inequality with (4.4) we obtain (4.1).

Denote by $T_{j}=\left[c_{j}, a_{j}\right]$ and $R_{j}=\left[b_{j}, d_{j}\right]$ the components of $E$ adjoint to $J_{j}$ and let

$$
S_{j}^{*}:=\phi_{ \pm}\left(S_{j}^{ \pm}\right), \quad T_{j}^{*}:=\phi_{ \pm}\left(T_{j}\right), \quad R_{j}^{*}:=\phi_{ \pm}\left(R_{j}\right)
$$

Lemma 16 The following holds:

$$
\begin{aligned}
\left|S_{j}^{*}\right| & \preceq\left|T_{j}^{*}\right|, \\
\left|S_{j}^{*}\right| & \preceq\left|R_{j}^{*}\right|, \\
\left|S_{j}^{*}\right| & \asymp\left|J_{j}\right| .
\end{aligned}
$$

Proof. By virtue of (1.5), (2.2), and (2.3) for

$$
\Gamma_{1}=\Gamma\left(a_{j}, b_{j} ; c_{j}, \infty ; G_{ \pm}\right), \quad \Gamma_{2}=\Gamma\left(a_{j}, b_{j} ; c_{j}, \infty ; \mathbf{C}\right)
$$


we have

$$
\begin{aligned}
1 & \preceq \frac{1}{2 \pi} \log \frac{b_{j}-c_{j}}{b_{j}-a_{j}} \leq m\left(\Gamma_{2}\right) \leq m\left(\Gamma_{1}\right) \\
& =m\left(\phi_{ \pm}\left(\Gamma_{1}\right)\right) \leq \frac{1}{\pi} \log \frac{\left|S_{j}^{*}\right|+\left|T_{j}^{*}\right|}{\left|S_{J}^{*}\right|}+2
\end{aligned}
$$

from which (4.6) follows.

The same reasoning applies to the proof of (4.7).

In order to prove (4.8), for sufficiently large $R$ consider the points $R \in E, t_{R}:=$ $\phi_{ \pm}(R)$ and families of curves

$$
\begin{gathered}
\Gamma_{3}=\Gamma\left(a_{j}, b_{j} ; R, \infty ; G_{ \pm}\right), \quad \Gamma_{4}=\Gamma\left(a_{j}, b_{j} ; R, \infty ; \mathbf{H}_{ \pm}\right), \\
\Gamma_{5}=\left\{\gamma_{a_{j}}\left(r, G_{ \pm}\right):\left|J_{j}\right|<r<R-a_{j}\right\} .
\end{gathered}
$$

According to (2.3) we obtain

$$
\begin{gathered}
\frac{1}{\pi} \log \frac{t_{R}-\phi_{ \pm}\left(a_{j}\right)}{\left|S_{j}^{*}\right|} \leq m\left(\phi_{ \pm}\left(\Gamma_{3}\right)\right) \leq \frac{1}{\pi} \log \frac{t_{R}-\phi_{ \pm}\left(a_{j}\right)}{\left|S_{j}^{*}\right|}+2 \\
m\left(\Gamma_{3}\right) \leq m\left(\Gamma_{4}\right) \leq \frac{1}{\pi} \log \frac{R-a_{j}}{\left|J_{j}\right|}+2 .
\end{gathered}
$$

Moreover, using the assumption (1.4) and reasoning as in the proof of (4.5) we have

$$
m\left(\Gamma_{3}\right) \geq m\left(\Gamma_{5}\right) \geq \frac{1}{\pi} \log \frac{R-a_{j}}{\left|J_{j}\right|}-C_{2} .
$$

The inequalities (4.9)-(4.11) yield

$$
\frac{\left|S_{j}^{*}\right|}{\left|J_{j}\right|} \asymp \frac{t_{R}-\phi_{ \pm}\left(a_{j}\right)}{R-a_{j}} .
$$

Letting $R \rightarrow \infty$ and applying (4.1) we obtain (4.8).

For $x \in E, \zeta \in \overline{G_{ \pm}}$, and $\sigma>0$ set

$$
\begin{gathered}
\zeta_{\sigma}^{ \pm}:=\psi_{ \pm}\left(\phi_{ \pm}(\zeta) \pm \frac{i}{\sigma}\right), \\
L_{\sigma}^{ \pm}:=\left\{\zeta \in G_{ \pm}: \Im \phi_{ \pm}(\zeta)= \pm \frac{1}{\sigma}\right\}, \quad d_{\sigma}(x):=d\left(x, L_{\sigma}^{ \pm} \cap \mathbf{H}_{ \pm}\right) .
\end{gathered}
$$


Lemma 17 For $x \in E, \zeta \in \mathbf{H}_{ \pm} \cap D\left(x, d_{\sigma}(x)\right)$, and $\sigma \geq 1$ we have

$$
\begin{aligned}
\left|x_{\sigma}^{ \pm}-x\right| & \preceq d_{\sigma}(x), \\
\left|\zeta_{\sigma}^{ \pm}-\zeta\right| & \asymp d_{\sigma}(x), \\
\left|\zeta_{\sigma}^{ \pm}-x\right| & \succeq d_{\sigma}(x) .
\end{aligned}
$$

Proof. According to (2.24), in order to prove (4.12) we can assume that $d_{\sigma}(x)<$ $r_{x}\left(x_{\sigma}^{ \pm}, G_{ \pm}\right)=: B$. Consider

$$
\Gamma_{1}=\Gamma\left(x, z ; x_{\sigma}^{ \pm}, \infty ; G_{ \pm}\right), \quad \Gamma_{2}=\left\{C(x, r): d_{\sigma}(x)<r<B\right\},
$$

where $z \in L_{\sigma}^{ \pm} \cap \overline{\mathbf{H}_{ \pm}}$is such that $|x-z|=d_{\sigma}(x)$. By virtue of (2.1) and (2.4)

$$
\frac{1}{2 \pi} \log \frac{B}{d_{\sigma}(x)}=m\left(\Gamma_{2}\right) \leq m\left(\Gamma_{1}\right)=m\left(\phi_{ \pm}\left(\Gamma_{1}\right)\right) \leq 2,
$$

which, together with (2.24), implies (4.12).

The inequality (4.13) follows immediately from Lemma 7 and (4.12).

Furthermore, by Lemma 6

$$
\left|\zeta_{\sigma}^{ \pm}-\zeta\right| \preceq d\left(\zeta_{\sigma}^{ \pm}, L^{ \pm}\right) \leq\left|\zeta_{\sigma}^{ \pm}-x\right|,
$$

which gives (4.14) when combined with (4.13).

Lemma 18 Let $x \in E, \zeta \in \mathbf{H}_{ \pm},|\zeta-x| \geq d_{\sigma}(x), \sigma \geq 1$. Then

$$
\left|\frac{\zeta_{\sigma}^{ \pm}-\zeta}{\zeta_{\sigma}^{ \pm}-x}\right| \preceq\left|\frac{d_{\sigma}(x)}{\zeta-x}\right|^{1 / 4}
$$

Proof. Let $t=\phi_{ \pm}(x), \tau^{ \pm}:=\phi_{ \pm}(\zeta), \tau_{\sigma}^{ \pm}:=\tau^{ \pm} \pm i / \sigma$. Let $\Gamma_{1}$ be the family of all closed curves in $G_{ \pm}$which separate $\zeta$ and $\zeta_{\sigma}^{ \pm}$from $L^{ \pm}$. Consider

$$
\Gamma_{2}=\Gamma\left(\zeta, \zeta_{\sigma}^{ \pm} ; x, \infty ; G_{ \pm}\right) \cup \Gamma_{1}, \quad \Gamma_{3}=\Gamma\left(x, x_{\sigma}^{ \pm} ; \zeta, \infty ; G_{ \pm}\right) .
$$

According to (2.2), (2.5), and (4.15) we have

$$
m\left(\Gamma_{2}\right) \leq \frac{1}{\pi} \log \left|\frac{\zeta_{\sigma}^{ \pm}-x}{\zeta_{\sigma}^{ \pm}-\zeta}\right|+C_{3} .
$$

Moreover, setting

$$
\Gamma_{4}=\left\{C\left(\tau_{\sigma}^{ \pm}, r\right): \frac{1}{\sigma}<r<\left|\tau_{\sigma}^{ \pm}-t\right|\right\}
$$


by virtue of (2.1) we obtain

$$
m\left(\Gamma_{2}\right)=m\left(\phi_{ \pm}\left(\Gamma_{2}\right)\right) \geq m\left(\Gamma_{4}\right)=\frac{1}{2 \pi} \log \sigma\left|\tau_{\sigma}^{ \pm}-t\right|
$$

which, together with (4.17), yields

$$
\left|\frac{\zeta_{\sigma}^{ \pm}-\zeta}{\zeta_{\sigma}^{ \pm}-x}\right| \preceq\left(\sigma\left|\tau_{\sigma}^{ \pm}-t\right|\right)^{-1 / 2} .
$$

Next, setting $\Gamma_{5}=\Gamma\left(x, x_{\sigma}^{ \pm} ; \zeta, \infty ; \mathbf{C}\right)$ and applying $(2.2)$ we have

$$
m\left(\Gamma_{3}\right) \geq m\left(\Gamma_{5}\right) \geq \frac{1}{2 \pi} \log \left|\frac{x-\zeta}{x-x_{\sigma}^{ \pm}}\right| .
$$

Furthermore, since by (2.19) $\left|\tau^{ \pm}-t\right| \succeq 1 / \sigma$ according to (2.3) and (2.4) we obtain

$$
m\left(\phi_{ \pm}\left(\Gamma_{3}\right)\right) \leq \frac{1}{\pi} \log \sigma\left|\tau_{\sigma}^{ \pm}-t\right|+C_{4}
$$

which, together with (4.19), implies that

$$
\left|\frac{x-x_{\sigma}^{ \pm}}{x-\zeta}\right| \succeq\left(\sigma\left|\tau_{\sigma}^{ \pm}-t\right|\right)^{-2}
$$

Comparing (4.12), (4.18), and (4.20) we obtain (4.16).

Lemma 19 Let $z_{1}, z_{2} \in \mathbf{H}_{ \pm}$be such that

$$
\Re z_{1}=\Re z_{2}=x \in E, \quad \Im z_{2}>\Im z_{1},
$$

and let $t=\phi_{ \pm}(x), w_{k}^{ \pm}=\phi_{ \pm}\left(z_{k}\right), k=1,2$. Then

$$
\begin{aligned}
&\left|w_{k}^{ \pm}-t\right| \preceq\left|\Im w_{k}^{ \pm}\right|, \\
&\left|\frac{w_{2}^{ \pm}-t}{w_{1}^{ \pm}-t}\right| \preceq\left|\frac{z_{2}-x}{z_{1}-x}\right|, \\
&\left|w_{1}^{ \pm}-t\right| \preceq\left|w_{2}^{ \pm}-t\right| .
\end{aligned}
$$

Proof. Let $w_{k}^{ \pm}=x_{k} \pm i y_{k}$. If $\left|t-x_{k}\right|>y_{k}$ consider

$$
\begin{gathered}
\Gamma_{1}=\Gamma_{1, k}=\left\{C\left(x_{k}, r\right) \cap \mathbf{H}: y_{k}<r<\left|t-x_{k}\right|\right\}, \\
\Gamma_{2}=\Gamma_{2, k}=\Gamma\left(z_{k} ; x, \infty ; G_{ \pm}\right), \quad \Gamma_{3}=\Gamma_{3, k}=\Gamma\left(z_{k} ; x, \infty ; \mathbf{H}_{ \pm}\right) .
\end{gathered}
$$


Since, by (2.1) and (2.6),

$$
\frac{1}{\pi} \log \frac{\left|t-x_{k}\right|}{y_{k}}=m\left(\Gamma_{1}\right)=m\left(\psi_{ \pm}\left(\Gamma_{1}\right)\right) \leq m\left(\Gamma_{2}\right) \leq m\left(\Gamma_{3}\right) \leq 2,
$$

we have $\left|t-x_{k}\right| \preceq y_{k}$, which establishes the formula (4.21).

Moreover, let

$$
\Gamma_{4}=\Gamma\left(x, z_{1} ; z_{2}, \infty ; G_{ \pm}\right), \quad \Gamma_{5}=\Gamma\left(x, z_{1} ; z_{2}, \infty ; \mathbf{H}_{ \pm}\right) .
$$

According to (2.3)

$$
\begin{aligned}
\frac{1}{\pi} \log \left|\frac{w_{2}^{ \pm}-t}{w_{1}^{ \pm}-t}\right| & \leq m\left(\phi_{ \pm}\left(\Gamma_{4}\right)\right)=m\left(\Gamma_{4}\right) \\
& \leq m\left(\Gamma_{5}\right) \leq \frac{1}{\pi} \log \left|\frac{z_{2}-x}{z_{1}-x}\right|+2,
\end{aligned}
$$

which implies (4.22).

Next, if $\left|t-w_{1}^{ \pm}\right|>\left|t-w_{2}^{ \pm}\right|$we consider

$$
\begin{gathered}
\Gamma_{6}=\Gamma\left(x, z_{2} ; z_{1}, \infty ; G_{ \pm}\right), \quad \Gamma_{7}=\Gamma\left(x, z_{2} ; z_{1}, \infty ; \mathbf{H}_{ \pm}\right), \\
\Gamma_{8}=\left\{C(t, r) \cap \mathbf{H}_{ \pm}:\left|t-w_{2}^{ \pm}\right|<r<\left|t-w_{1}^{ \pm}\right|\right\} .
\end{gathered}
$$

By virtue of (2.1) and (2.4) we have

$$
\begin{aligned}
\frac{1}{\pi} \log \left|\frac{t-w_{1}^{ \pm}}{t-w_{2}^{ \pm}}\right| & =m\left(\Gamma_{8}\right) \leq m\left(\phi_{ \pm}\left(\Gamma_{6}\right)\right) \\
& =m\left(\Gamma_{6}\right) \leq m\left(\Gamma_{7}\right) \leq 2,
\end{aligned}
$$

from which (4.23) follows.

Lemma 20 For $z \in \mathbf{H}_{ \pm}$with $d(z, E) \leq 3$,

$$
\begin{aligned}
\left|\Im \phi_{ \pm}(z)\right| & \preceq 1, \\
\left|\phi_{ \pm}^{\prime}(z)\right| & \succeq 1 .
\end{aligned}
$$

Proof. Let $w=w^{ \pm}=\phi_{ \pm}(z)$ and let $R \in E$ be sufficiently large. Let $x \in E$ satisfy $|z-x|=d(z, E), t_{R}=\phi_{ \pm}(R)$, and let

$$
\Gamma_{1}=\Gamma\left(z ; R, \infty ; G_{ \pm}\right), \quad \Gamma_{2}=\left\{\gamma_{x}\left(r, G_{ \pm}\right): 3<r<R-x\right\} .
$$


By virtue of (2.5) we have

$$
m\left(\Gamma_{1}\right)=m\left(\phi_{ \pm}\left(\Gamma_{1}\right)\right) \leq \frac{1}{\pi} \log \frac{t_{R}-\Re w}{\Im w}+2 .
$$

Moreover, reasoning as in the proof of (4.5) we obtain

$$
m\left(\Gamma_{1}\right) \geq m\left(\Gamma_{2}\right)=\int_{3}^{R-x} \frac{d r}{\left|\gamma_{x}\left(r, G_{ \pm}\right)\right|} \geq \frac{1}{\pi} \log (R-x)-C_{5} .
$$

Therefore, (4.26) and (4.27) yield

$$
|\Im w| \preceq \frac{t_{R}-\Re w}{R-x} .
$$

Applying (4.1) and letting $R \rightarrow \infty$ we have (4.24).

Next, let $w=u \pm i v, \xi=\xi_{ \pm}=\psi_{ \pm}(u)$. Since by $(2.21)|z-\xi| \asymp d(z, E)$, according to Lemma 5 for $\Gamma_{3}=\Gamma\left(\xi, z ; R, \infty ; G_{ \pm}\right)$we obtain

$$
\begin{aligned}
m\left(\Gamma_{3}\right) & \leq m_{\xi}\left(|\xi-z|,|\xi-R| ; G_{ \pm}\right)+C_{6} \\
& \leq \frac{1}{\pi} \log \left|\frac{\xi-R}{\xi-z}\right|+C_{6} .
\end{aligned}
$$

In the opposite direction, by virtue of (2.3)

$$
m\left(\Gamma_{3}\right)=m\left(\phi_{ \pm}\left(\Gamma_{3}\right)\right) \geq \frac{1}{\pi} \log \frac{t_{R}-u}{v}
$$

Therefore,

$$
\frac{v}{d(z, E)} \succeq \frac{t_{R}-u}{|R-\xi|}
$$

Letting $R \rightarrow \infty$ and taking into account (4.1) we have

$$
\frac{|\Im w|}{d(z, E)} \succeq 1
$$

To complete the proof of (4.25) we have to use the immediate consequence of the Koebe 1/4-Lemma, i.e., the inequality

$$
\left|\phi_{ \pm}^{\prime}(z)\right| \geq \frac{1}{4} \frac{|\Im w|}{d\left(z, L^{ \pm}\right)} \geq \frac{1}{4} \frac{|\Im w|}{d(z, E)}
$$

(see [7, p. 58]). 
Lemma 21 Let $x \in E, 0<\delta \leq 1, t=\phi_{ \pm}(x), \tilde{t}_{\delta}^{ \pm}=\phi_{ \pm}(x \pm i \delta)$. Then

$$
\left|t-\tilde{t}_{\delta}^{ \pm}\right| \succeq \delta
$$

Proof. Let $R$ be sufficiently large and let $t_{R}:=\phi_{ \pm}(R)$. According to (2.3) for

$$
\Gamma_{1}=\Gamma\left(x, x \pm i \delta ; R, \infty ; G_{ \pm}\right), \quad \Gamma_{2}=\Gamma\left(x, x \pm i \delta ; R, \infty ; \mathbf{H}_{ \pm}\right)
$$

we have

$$
\begin{aligned}
\frac{1}{\pi} \log \frac{t_{R}-t}{\left|\tilde{t}_{\delta}^{ \pm}-t\right|} & \leq m\left(\phi_{ \pm}\left(\Gamma_{1}\right)\right)=m\left(\Gamma_{1}\right) \leq m\left(\Gamma_{2}\right) \\
& \leq \frac{1}{\pi} \log \frac{R-x}{\delta}+2
\end{aligned}
$$

which yields

$$
\frac{\delta}{\left|\tilde{t}_{\delta}^{ \pm}-t\right|} \preceq \frac{R-x}{t_{R}-t} .
$$

Letting $R \rightarrow \infty$ and applying (4.1) we obtain (4.28).

Next, we improve the inequality (4.28) for points $x \in E$ close to the components $J_{j}=\left(a_{j}, b_{j}\right)$ of $E^{*}$.

Lemma 22 Under the assumptions and notation of Lemma 21 for $x \in E$ such that $d\left(x, J_{j}\right) \leq C_{5}\left|J_{j}\right| / 2$, where $C_{5}$ is the constant from (1.5), the following inequalities hold.

(i) If $\delta<d\left(x, J_{j}\right)$, then

$$
\left|t-\tilde{t}_{\delta}^{ \pm}\right| \succeq\left(\frac{\left|J_{j}\right|}{d\left(x, J_{j}\right)}\right)^{1 / 2} \delta .
$$

(ii) If $d\left(x, J_{j}\right) \leq \delta \leq\left|J_{j}\right|$, then

$$
\left|t-\tilde{t}_{\delta}^{ \pm}\right| \succeq\left|J_{j}\right|^{1 / 2} \delta^{1 / 2}
$$

Proof. Let $S_{j}^{*}:=\phi_{ \pm}\left(S_{j}^{ \pm}\right)=\left(a_{j}^{*}, b_{j}^{*}\right)$. Without loss of generality we can assume that $x \geq b_{j}$.

(i) First, we claim that

$$
\left|t-b_{j}^{*}\right| \succeq\left(x-b_{j}\right)^{1 / 2}\left|J_{j}\right|^{1 / 2} .
$$


Indeed, by Lemmas 2 and 5 for the module of the family $\Gamma_{1}=\Gamma\left(x, b_{j} ; a_{j}, \infty ; G_{ \pm}\right)$ we have

$$
m\left(\Gamma_{1}\right) \leq m_{x}\left(x-b_{j}, x-a_{j} ; G_{ \pm}\right)+C_{7} \leq \frac{1}{2 \pi} \log \frac{\left|J_{j}\right|}{x-b_{j}}+C_{8} .
$$

Moreover, according to $(2.3)$

$$
m\left(\phi_{ \pm}\left(\Gamma_{1}\right)\right) \geq \frac{1}{\pi} \log \frac{t-a_{j}^{*}}{t-b_{j}^{*}} \geq \frac{1}{\pi} \log \frac{\left|S_{j}^{*}\right|}{t-b_{j}^{*}} .
$$

Therefore, (4.8), (4.32), and (4.33) imply (4.31).

Next, by virtue of (2.3) for

$$
\Gamma_{2}=\Gamma\left(x, x \pm i \delta ; b_{j}, \infty ; G_{ \pm}\right), \quad \Gamma_{3}=\Gamma\left(x, b_{j} ; a_{j}, \infty ; \mathbf{H}_{ \pm}\right)
$$

we have

$$
\begin{aligned}
\frac{1}{\pi} \log \frac{t-b_{j}^{*}}{\left|t-\tilde{t}_{\delta}^{ \pm}\right|} & \leq m\left(\phi_{ \pm}\left(\Gamma_{2}\right)\right)=m\left(\Gamma_{2}\right) \leq m\left(\Gamma_{3}\right) \\
& \leq \frac{1}{\pi} \log \frac{x-b_{j}}{\delta}+2,
\end{aligned}
$$

which gives (4.29) when combined with (4.31).

(ii) By Lemmas 2 and 5 for

$$
\Gamma_{4}=\Gamma\left(x, x \pm i \delta ; a_{j}, \infty ; G_{ \pm}\right)
$$

we obtain

$$
\begin{aligned}
m\left(\Gamma_{4}\right) & \leq m_{x}\left(\delta, x-a_{j} ; G_{ \pm}\right)+C_{9} \\
& \leq \frac{1}{2 \pi} \log \frac{\left|J_{j}\right|}{\delta}+C_{10} .
\end{aligned}
$$

On the other hand, by virtue of (2.3) and (4.8)

$$
m\left(\phi_{ \pm}\left(\Gamma_{4}\right)\right) \geq \frac{1}{\pi} \log \frac{t-a_{j}^{*}}{\left|t-\tilde{t}_{\delta}^{ \pm}\right|} \geq \frac{1}{\pi} \log \frac{\left|J_{j}\right|}{\left|t-\tilde{t}_{\delta}^{ \pm}\right|}-C_{11} .
$$

The inequalities (4.34) and (4.35) imply (4.30).

In the proof of Theorem 2 we need the following immediate consequence of Lemmas 13, 14, 21, and 22. Let $x \in E, 0<\delta \leq 1, w=\phi(x), w_{\delta}=\phi(x+i \delta), t=$ $\phi_{ \pm}(x), \tilde{t}_{\delta}^{ \pm}=\phi_{ \pm}(x \pm i \delta)$. Then

$$
\left|w-w_{\delta}\right| \preceq\left|t-\tilde{t}_{\delta}^{ \pm}\right| .
$$




\section{The Extension Operator for an Antiderivative}

Let $f \in B C_{\omega}^{*}(E)$. We continuously extend $f$ to $\mathbf{R}$ such that on any component $J_{j}$ of $E^{*}$ it is a linear function. By the Lagrange formula for $x \in J_{j}$,

$$
|f(x)|=\left|f\left(b_{j}\right) \frac{x-a_{j}}{b_{j}-a_{j}}-f\left(a_{j}\right) \frac{x-b_{j}}{b_{j}-a_{j}}\right| \leq\|f\|_{C(E)} .
$$

This clearly forces

$$
\|f\|_{C(\mathbf{R})}=\|f\|_{C(E)} .
$$

Furthermore, let $x_{1}, x_{2} \in E$ and $\xi_{1}, \xi_{2} \in \mathbf{R}$ satisfy $x_{1} \leq \xi_{1}<\xi_{2} \leq x_{2}$. For $k=1,2$ consider points $\nu_{k} \in E \cap\left[x_{1}, x_{2}\right]$ such that $\left|\xi_{k}-\nu_{k}\right|=d\left(\xi_{k}, E\right)$. Then

$$
\begin{aligned}
\left|f\left(\xi_{2}\right)-f\left(\xi_{1}\right)\right| & \leq\left|f\left(\xi_{2}\right)-f\left(\nu_{2}\right)\right|+\left|f\left(\nu_{2}\right)-f\left(\nu_{1}\right)\right|+\left|f\left(\nu_{1}\right)-f\left(\xi_{1}\right)\right| \\
& \leq 3 \omega\left(\operatorname{diam} \phi\left(\left[x_{1}, x_{2}\right]\right)\right) .
\end{aligned}
$$

Consider

$$
F(x):=\int_{0}^{x} f(t) d t \quad(x \in E) .
$$

Our next objective is to continuously extend $F$ from $E$ to $\mathbf{C}$. The procedure described below is a modification of the corresponding constructions from [20] and [9] (see also [7, pp. 13-15]).

Lemma 23 ([20, Chapter VI]). There exist a collection of closed squares $Q_{k} \subset$ $\mathbf{C} \backslash E, k \in \mathbf{N}$ with sides parallel to the coordinate axes, and a set of infinitely differentiable (with respect to $x$ and $y$ ) functions $\mu_{k}(z)=\mu_{k}(x+i y)$ satisfying the following properties.

(i) $\cup_{k} Q_{k}=\mathbf{C} \backslash E$.

(ii) $\operatorname{diam} Q_{k} \leq 2 d\left(Q_{k}, E\right) \leq 8 \operatorname{diam} Q_{k}$.

(iii) Each point $z \in \mathbf{C} \backslash E$ is contained in at most 144 of the squares $Q_{k}$.

(iv) $\sum_{k} \mu_{k}(z)=1 \quad(z \in \mathbf{C} \backslash E)$.

(v) $\mu_{k}(z)=0 \quad\left(z \in \mathbf{C} \backslash Q_{k}\right)$.

(vi) For $r, l \in \mathbf{N}_{0}$ such that $r+l \leq 1$ we have

$$
\left|\frac{\partial^{r+l} \mu_{k}(z)}{\partial x^{r} \partial y^{l}}\right| \preceq\left(\operatorname{diam} Q_{k}\right)^{-r-l}
$$


Let for $x \in E$ and $z \in \mathbf{C}$,

$$
P_{x}(z)=P_{x, f, E}(z):=F(x)+f(x)(z-x) .
$$

Denote by $x_{k} \in E$ a point with the property $d\left(Q_{k}, E\right)=d\left(Q_{k}, x_{k}\right)$. The extension operator $\mathcal{E}$ is defined as follows:

$$
\mathcal{E} F(z):= \begin{cases}\sum_{k}^{\prime} P_{x_{k}}(z) \mu_{k}(z), & \text { if } z \in \mathbf{C} \backslash E \\ F(z), & \text { if } z \in E\end{cases}
$$

where $\sum_{k}^{\prime}$ means the sum taken over only those squares $Q_{k}$ for which $d\left(Q_{k}, E\right)<$ 1. Next, we clarify the difference between the sum $\Sigma_{k}^{\prime}$ and the complete sum $\Sigma_{k}$. If $d(z, E)<1$, then $\Sigma_{k}^{\prime}=\Sigma_{k}$. If $d(z, E)>3$, then for every square $Q_{k} \ni z$, by Lemma 23(ii),

$$
3<d(z, E) \leq \operatorname{diam} Q_{k}+d\left(Q_{k}, E\right) \leq 3 d\left(Q_{k}, E\right),
$$

i.e., $\Sigma_{k}^{\prime}$ does not have any terms at all.

For brevity, we also use the same notation $F$ for the extension $\mathcal{E} F$. The remark above yields

$$
F(z)=0 \quad(z \in \mathbf{C}, d(z, E) \geq 3) .
$$

Lemma 24 The function $F$ possesses the following properties.

(i) $F$ is continuous in $\mathbf{C}$.

(ii) $F(z)=F(x+i y)$ is infinitely differentiable (with respect to $x$ and $y$ ) in $\mathbf{C} \backslash E$ and for $z \in \mathbf{C} \backslash E$ we have

$$
\left|\frac{\partial F(z)}{\partial \bar{z}}\right| \preceq \begin{cases}\omega\left(\mid \phi\left(z^{\prime}\right)-\phi\left(z^{\prime}+i d(z, E) \mid\right),\right. & \text { if } d(z, E)<1, \\ |z||| f \|_{C(E)}, & \text { if } 1 \leq d(z, E) \leq 3,\end{cases}
$$

where $z^{\prime} \in E$ satisfies $d(z, E)=\left|z-z^{\prime}\right|$.

(iii) For $x \in E, z \in \mathbf{C},|z-x|=\delta, 0<\delta \leq 1$,

$$
\left|F(z)-P_{x}(z)\right| \preceq \omega(|\phi(x)-\phi(x+i \delta)|) \delta .
$$

Proof. Let $z \in Q_{k}$, by Lemma 23(iii) the number of such squares is at most 144. According to Lemma 23(ii) for $\delta_{k}:=$ diam $Q_{k}$ we obtain

$$
\frac{\delta_{k}}{2} \leq d\left(Q_{k}, E\right) \leq d(z, E) \leq \delta_{k}+d\left(Q_{k}, E\right) \leq 5 \delta_{k} .
$$


Furthermore, if $z_{k} \in Q_{k}$ and $x_{k} \in E$ are such that $\left|x_{k}-z_{k}\right|=d\left(Q_{k}, E\right)$, then

$$
\left|z^{\prime}-x_{k}\right| \leq\left|z^{\prime}-z\right|+\left|z-z_{k}\right|+\left|z_{k}-x_{k}\right| \leq 11 \delta_{k} \leq 22 d(z, E) .
$$

(i) We only need to show that for any $x \in E$,

$$
\lim _{\mathbf{C} \backslash E \ni z \rightarrow x} F(z)=F(x) .
$$

By virtue of Lemma 23(iv) for $z \in \mathbf{C} \backslash E$ with $|z-x|<1$ we have

$$
|F(z)-F(x)|=\sum_{k}\left(P_{x_{k}}(z)-F(x)\right) \mu_{k}(z)
$$

which implies (5.6).

(ii) Consider the linear functions

$$
L_{k}(\zeta):=P_{x_{k}}(\zeta)-P_{z^{\prime}}(\zeta) \quad(\zeta \in \mathbf{C})
$$

and points $u_{k} \in E$ such that

$$
\left|u_{k}-z^{\prime}\right| \asymp\left|u_{k}-x_{k}\right| \asymp d(z, E) .
$$

Since, by the Mean Value Theorem for $\xi_{1}, \xi_{2} \in E$,

$$
\left|P_{\xi_{1}}\left(\xi_{2}\right)-F\left(\xi_{2}\right)\right|=\left|f\left(\xi_{1}\right)-\left(\Re f\left(c_{1}\right)+i \Im f\left(c_{2}\right)\right)\right|\left|\xi_{2}-\xi_{1}\right|,
$$

for some $c_{1}$ and $c_{2}$ between $\xi_{1}$ and $\xi_{2},(1.10),(3.6),(5.2),(5.5)$, and Lemma 6 imply that

$$
\begin{aligned}
& \left|L_{k}\left(x_{k}\right)\right|=\left|F\left(x_{k}\right)-P_{z^{\prime}}\left(x_{k}\right)\right| \preceq \omega\left(\operatorname{diam} \phi\left(\left[x_{k}, z^{\prime}\right]\right)\right)\left|x_{k}-z^{\prime}\right| \\
& \preceq \omega\left(\left|\phi\left(z^{\prime}\right)-\phi\left(z^{\prime}+i d(z, E)\right)\right|\right) d(z, E), \\
& \left|L_{k}\left(u_{k}\right)\right| \leq \mid P_{x_{k}}\left(u_{k}\right)-F\left(u_{k}|+| F\left(u_{k}\right)-P_{z^{\prime}}\left(u_{k}\right) \mid\right. \\
& \preceq \omega\left(\operatorname{diam} \phi\left(\left[x_{k}, u_{k}\right]\right)\right)\left|x_{k}-u_{k}\right|+\omega\left(\operatorname{diam} \phi\left(\left[u_{k}, z^{\prime}\right]\right)\right)\left|u_{k}-z^{\prime}\right| \\
& \preceq \omega\left(\left|\phi\left(z^{\prime}\right)-\phi\left(z^{\prime}+i d(z, E)\right)\right|\right) d(z, E),
\end{aligned}
$$

where $\left[x_{k}, z^{\prime}\right],\left[x_{k}, u_{k}\right]$, and $\left[u_{k}, z^{\prime}\right]$ are the intervals of $\mathbf{R}$ between the appropriate points.

Moreover, by the Lagrange interpolation formula we obtain

$$
\begin{aligned}
\left|L_{k}(z)\right| & \leq\left|L_{k}\left(u_{k}\right)\right|\left|\frac{z-x_{k}}{u_{k}-x_{k}}\right|+\left|L_{k}\left(x_{k}\right)\right|\left|\frac{z-u_{k}}{x_{k}-u_{k}}\right| \\
& \preceq \omega\left(\left|\phi\left(z^{\prime}\right)-\phi\left(z^{\prime}+i d(z, E)\right)\right|\right) d(z, E) .
\end{aligned}
$$


Therefore, if $d(z, E)<1$ then Lemma 23(vi), (5.4), and (5.7) yield that

$$
\begin{aligned}
\left|\frac{\partial F(z)}{\partial \bar{z}}\right| & =\left|\frac{\partial}{\partial \bar{z}}\left(F(z)-P_{z^{\prime}}(z)\right)\right| \\
& =\left|\sum_{k} L_{k}(z) \frac{\partial \mu_{k}(z)}{\partial \bar{z}}\right| \preceq \omega\left(\left|\phi\left(z^{\prime}\right)-\phi\left(z^{\prime}+i d(z, E)\right)\right|\right) .
\end{aligned}
$$

Let $1 \leq d(z, E) \leq 3$. Since according to $(5.1)$

$$
|F(x)| \leq|x|\|f\|_{C(E)} \quad(x \in E)
$$

and, therefore, for the squares $Q_{k} \ni z$,

$$
\left|P_{x_{k}}(z)\right| \preceq|z||| f \|_{C(E)}
$$

by virtue of Lemma 23(vi) we obtain

$$
\left.\left|\frac{\partial F(z)}{\partial \bar{z}}\right|=\mid \sum_{k}^{\prime} P_{x_{k}}(z) \frac{\partial \mu_{k}(z)}{\partial \bar{z}}\right)|\preceq| z|| \mid f \|_{C(E)},
$$

which, together with (5.8), proves (ii).

(iii) Since $F$ is continuous in $\mathbf{C}$ we can assume that $z \in C(x, \delta) \backslash E$. For $Q_{k} \ni z$ consider the linear functions

$$
L_{k}^{*}(\zeta):=P_{x_{k}}(\zeta)-P_{x}(\zeta) \quad(\zeta \in \mathbf{C})
$$

and points $u_{k}^{*} \in E$ such that

$$
\left|u_{k}^{*}-x\right| \asymp\left|u_{k}^{*}-x_{k}\right| \asymp \delta .
$$

Repeating the reasoning from the proof of the part (ii) we obtain

$$
\left|L_{k}^{*}(z)\right| \preceq \omega(|\phi(x)-\phi(x+i \delta)|) \delta .
$$

Therefore, according to Lemma 23 we have

$$
\left|F(z)-P_{x}(z)\right|=\left|\sum_{k} L_{k}^{*}(z) \mu_{k}(z)\right| \preceq \omega(|\phi(x)-\phi(x+i \delta)|) \delta,
$$

and (iii) is proved. 


\section{Auxiliary Entire Functions}

In this section we discuss the construction of certain entire functions of exponential type. Our reasoning is influenced by [18] and [10, Chapter IX].

We start with well-known facts from the harmonic analysis (see, for details [1]). Let $g \in L(\mathbf{R})$, i.e.,

$$
\|g\|_{L(\mathbf{R})}:=\int_{\mathbf{R}}|g(x)| d x<\infty
$$

and let

$$
\hat{g}(t):=\frac{1}{\sqrt{2 \pi}} \int_{\mathbf{R}} g(x) e^{-i t x} d x \quad(t \in \mathbf{R})
$$

be the Fourier transform of $g$.

For $\sigma>0$ and $s \in \mathbf{N}_{0}$ such that $0 \leq s<99$ consider the function

$$
Q_{\sigma, s}(z):=\frac{\sin ^{100} \sigma z}{z^{100-s}} \quad(z \in \mathbf{C}) .
$$

Since $Q_{\sigma, s} \in E_{100 \sigma}$ and $\int_{\mathbf{R}}\left|Q_{\sigma, s}(x)\right|^{2} d x<\infty$ we have

$$
\hat{Q}_{\sigma, s}(t)=0 \quad(t \in \mathbf{R},|t| \geq 100 \sigma) .
$$

Moreover, for $t \in \mathbf{R}$,

$$
g_{\sigma, s}(t):=\int_{\mathbf{R}} g(x) Q_{\sigma, s}(t-x) d x=\int_{-100 \sigma}^{100 \sigma} \hat{g}(x) \hat{Q}_{\sigma, s}(x) e^{i t x} d x .
$$

Therefore, $g_{\sigma, s}$ can be extended to the entire function (for which we use the same notation). Since

$$
\|\hat{g}\|_{C(\mathbf{R})} \leq \frac{1}{\sqrt{2 \pi}}\|g\|_{L(\mathbf{R})}
$$

and

$$
\left\|\hat{Q}_{\sigma, s}\right\|_{C(\mathbf{R})} \leq \frac{1}{\sqrt{2 \pi}}\left\|Q_{\sigma, s}\right\|_{L(\mathbf{R})} \leq C_{1}(\sigma),
$$

for $z \in \mathbf{C}$ we have

$$
\left|g_{\sigma, s}(z)\right| \leq\left. C_{2}(\sigma)|| g\right|_{L(\mathbf{R})} \exp (100 \sigma|\Im z|)
$$

(cf. [1, p. 134]).

Let $Q_{\sigma}:=Q_{\sigma, 0}$. According to (6.1) the function

$$
\begin{aligned}
g_{\sigma, s}^{*}(t) & :=\int_{\mathbf{R}} g(x) x^{s} Q_{\sigma}(t-x) d x \\
& =\sum_{l=0}^{s} \frac{(-1)^{l} s !}{l !(s-l) !} t^{s-l} g_{\sigma, l}(t) \quad(t \in \mathbf{R})
\end{aligned}
$$


belongs to $E_{100 \sigma}$. Moreover, for $|z| \geq 1$,

$$
\left|g_{\sigma, s}^{*}(z)\right| \leq\left. C_{3}(\sigma)|| g\right|_{L(\mathbf{R})}|z|^{s} \exp (100 \sigma|\Im z|) .
$$

Let $f \in B C_{\omega}^{*}(E)$ and let $F$ be defined as in Section 5. For fixed $z_{0}:=4 i$ let

$$
\begin{gathered}
F_{0}(z):=\frac{F(z)}{\left(z-z_{0}\right)^{3}} \quad(z \in \mathbf{C}), \\
\lambda_{0}(z):=\frac{1}{\pi} \frac{\partial F_{0}(z)}{\partial \bar{z}}=\frac{1}{\pi\left(z-z_{0}\right)^{3}} \frac{\partial F(z)}{\partial \bar{z}} \quad(z \in \mathbf{C} \backslash E), \\
\lambda^{ \pm}(w):= \begin{cases}\lambda_{0}\left(\psi_{ \pm}(w),\right. & \text { if } w \in \mathbf{H}_{ \pm}, \psi_{ \pm}(w) \in \mathbf{H}_{ \pm}, \\
0, & \text { if } w \in \mathbf{H}_{ \pm}, \psi_{ \pm}(w) \in \mathbf{H}_{\mp} .\end{cases}
\end{gathered}
$$

Consider the kernel

$$
K(t):=C\left(\frac{\sin t}{t}\right)^{100} \quad(t \in \mathbf{R}),
$$

where

$$
C:=\left(\int_{\mathbf{R}}\left(\frac{\sin t}{t}\right)^{100} d t\right)^{-1} .
$$

For $\zeta \in G_{ \pm}, \sigma>0$, and $t \in \mathbf{R}$ set

$$
\zeta_{\sigma, t}^{ \pm}:=\psi_{ \pm}\left(\phi_{ \pm}(\zeta) \pm \frac{i}{\sigma}-t\right) .
$$

Furthermore, for $z \in \mathbf{C} \backslash G_{ \pm}$set

$$
e_{\sigma}^{ \pm}(z):=\sigma \int_{\mathbf{R}} K(\sigma t) \int_{\mathbf{H}_{ \pm}} \lambda_{0}(\zeta) \sum_{j=0}^{8} \frac{\left(\zeta_{\sigma, t}^{ \pm}-\zeta\right)^{j}}{\left(\zeta_{\sigma, t}^{ \pm}-z\right)^{j+1}} d m(\zeta) d t,
$$

where $d m(\zeta)$ means integration with respect to the two-dimensional Lebesgue measure (area).

Lemma 25 The function $e_{\sigma}^{ \pm}$can be extended to the entire function belonging to $E_{C_{4} \sigma}$.

Proof. By virtue of (4.24) and (5.3) for $z \in \mathbf{C} \backslash G_{ \pm}$,

$$
\begin{aligned}
e_{\sigma}^{ \pm}(z)= & \sigma \int_{\mathbf{R}} K(\sigma t) \int_{\mathbf{R}} \int_{0}^{v_{0}} \lambda^{ \pm}(w)\left|\psi_{ \pm}^{\prime}(w)\right|^{2} \\
& \sum_{j=0}^{8} \frac{\left(\psi_{ \pm}\left(w \pm \frac{i}{\sigma}-t\right)-\psi_{ \pm}(w)\right)^{j}}{\left(\psi_{ \pm}\left(w \pm \frac{i}{\sigma}-t\right)-z\right)^{j+1}} d v d u d t
\end{aligned}
$$


where $w=u \pm i v$ and $0<v_{0} \preceq 1$.

For $u \in \mathbf{R}, 0<v<v_{0}$, and $0 \leq l \leq j \leq 8$ consider the functions

$$
\begin{gathered}
\lambda_{v}^{ \pm}(u):=\lambda^{ \pm}(u \pm i v)\left|\psi_{ \pm}^{\prime}(u \pm i v)\right|^{2}, \\
h^{ \pm}(z)=h_{\sigma, v, l, j}^{ \pm}(z) \\
:=\int_{\mathbf{R}} K(\sigma t) \int_{\mathbf{R}} \lambda_{v}^{ \pm}(u) \frac{\psi_{ \pm}\left(u \pm i v \pm \frac{i}{\sigma}-t\right)^{l} \psi_{ \pm}(u \pm i v)^{j-l}}{\left(\psi_{ \pm}\left(u \pm i v \pm \frac{i}{\sigma}-t\right)-z\right)^{j+1}} d u d t \\
=\int_{\mathbf{R}} K(\sigma t) \int_{\mathbf{R}} \lambda_{v}^{ \pm}(\xi+t) \frac{\psi_{ \pm}\left(\xi \pm i v \pm \frac{i}{\sigma}\right)^{l} \psi_{ \pm}(t+\xi \pm i v)^{j-l}}{\left(\psi_{ \pm}\left(\xi \pm i v \pm \frac{i}{\sigma}\right)-z\right)^{j+1}} d \xi d t \\
=\int_{\mathbf{R}} \frac{\psi_{ \pm}\left(\xi \pm i v \pm \frac{i}{\sigma}\right)^{l}}{\left(\psi_{ \pm}\left(\xi \pm i v \pm \frac{i}{\sigma}\right)-z\right)^{j+1}} q^{ \pm}(\xi) d \xi,
\end{gathered}
$$

where

$$
\begin{aligned}
& q^{ \pm}(\xi):=\int_{\mathbf{R}} K(\sigma t) p^{ \pm}(\xi+t) d t \\
&=\int_{\mathbf{R}} K(\sigma(\eta-\xi)) r^{ \pm}(\eta)(\eta \pm i)^{8} d \eta \\
& p^{ \pm}(\xi):= \lambda_{v}^{ \pm}(\xi) \psi_{ \pm}(\xi \pm i v)^{j-l} \\
&= \lambda^{ \pm}(\xi \pm i v)\left|\psi_{ \pm}^{\prime}(\xi \pm i v)\right|^{2} \psi_{ \pm}(\xi \pm i v)^{j-l}, \\
& r^{ \pm}(\eta):=\frac{p^{ \pm}(\eta)}{(\eta \pm i)^{8}} .
\end{aligned}
$$

By (4.1) and Lemma 24(ii)

$$
\left|\lambda^{ \pm}(\xi \pm i v)\right| \preceq|| f \|_{C(E)}\left|\xi-z_{0}\right|^{-2},
$$

and according to (4.25) for $\xi \pm i v$ such that $\lambda^{ \pm}(\xi \pm i v) \neq 0$ we obtain

$$
\left|\psi_{ \pm}^{\prime}(\xi \pm i v)\right| \preceq 1 .
$$

Therefore, by virtue of (4.1) and (6.2) for $|\zeta| \geq 1$ we have

$$
\left|q^{ \pm}(\zeta)\right| \leq C_{5}(E, \sigma)\|f\|_{C(E)}|\zeta|^{8} \exp (100 \sigma|\Im \zeta|) .
$$

Notice that $h^{ \pm}$can be extended analytically from $\mathbf{C} \backslash G_{ \pm}$to $\mathbf{C}$ as follows. By (2.19) for $z \in \mathbf{C}$ with $|z| \geq 1$ we can find $r$ such that

$$
2|z| \leq\left|\psi_{ \pm}(w)\right| \preceq|z| \quad\left(w \in \overline{\mathbf{H}_{ \pm}} \cap C(r)\right) .
$$


According to (4.1) $r \leq C_{6}|z|$. Then the above mentioned extension is defined by the formula

$$
h^{ \pm}(z)=\int_{J_{r}} \frac{\psi_{ \pm}\left(\xi \pm i v \pm \frac{i}{\sigma}\right)^{l}}{\left(\psi_{ \pm}\left(\xi \pm i v \pm \frac{i}{\sigma}\right)-z\right)^{j+1}} q^{ \pm}(\xi) d \xi,
$$

where $J_{r}:=\left(C(r) \cap \mathbf{H}_{ \pm}\right) \cup(\mathbf{R} \backslash D(r))$. We may now differentiate 9 times to conclude that according to (4.1) and (6.3) for $0<v<v_{0}$,

$$
\left|\frac{d^{9}}{d z^{9}} h^{ \pm}(z)\right| \preceq \exp \left(100 C_{6} \sigma|z|\right) .
$$

Therefore,

$$
\left|h^{ \pm}(z)\right| \preceq \exp \left(C_{7} \sigma|z|\right) .
$$

Comparing the definitions of $e_{\sigma}^{ \pm}$and $h^{ \pm}$with the above inequality we have $e_{\sigma}^{ \pm} \in E_{C_{7} \sigma}$.

\section{Proof of Theorem 2}

Let $z_{0}=4 i$ and let functions $F, P_{x}, \lambda_{0}, e_{\sigma}^{ \pm}, e_{\sigma}:=e_{\sigma}^{+}+e_{\sigma}^{-}$be defined as in Sections 5 and 6. By (1.10) and Lemma 25 in order to prove (1.11) it is sufficient to show that for $\sigma>2$ and $x \in E$,

$$
\left|\frac{f(x)}{\left(x-z_{0}\right)^{3}}-e_{\sigma}^{\prime}(x)-\frac{3 e_{\sigma}(x)}{x-z_{0}}\right| \preceq\left|x-z_{0}\right|^{-3}\left(\frac{\|f\|_{C(E)}}{\sigma}+\omega\left(\frac{1}{\sigma}\right)\right) .
$$

Let for $\zeta \in G_{ \pm}$and $x \in E$,

$$
\begin{gathered}
R^{ \pm}(\zeta, x)=R_{\sigma, t}^{ \pm}(\zeta, x):=\sum_{j=0}^{8} \frac{\left(\zeta_{\sigma, t}^{ \pm}-\zeta\right)^{j}}{\left(\zeta_{\sigma, t}^{ \pm}-x\right)^{j+1}}, \\
R(\zeta, x)=R_{\sigma, t}(\zeta, x):=\sum_{ \pm} R^{ \pm}(\zeta, x), \\
d:=d_{\sigma}(x), \quad D:=D(x, d), \quad J:=C(x, d), \quad \Sigma:=\{z: d(z, E) \leq 3\} .
\end{gathered}
$$

Since for $x \in E$

$$
\begin{aligned}
\frac{f(x)}{\left(x-z_{0}\right)^{3}} & =\frac{1}{2 \pi i} \int_{J} \frac{F(\zeta)}{\left(\zeta-z_{0}\right)^{3}}\left(\frac{1}{(\zeta-x)^{2}}+\frac{3}{(\zeta-x)\left(x-z_{0}\right)}\right) d \zeta \\
& +\frac{1}{2 \pi i} \int_{J} \frac{P_{x}(\zeta)-F(\zeta)}{\left(\zeta-z_{0}\right)^{3}}\left(\frac{1}{(\zeta-x)^{2}}+\frac{3}{(\zeta-x)\left(x-z_{0}\right)}\right) d \zeta
\end{aligned}
$$


and according to the Green formula (cf. [7, p. 22])

$$
\begin{aligned}
& \frac{1}{2 \pi i} \int_{J} \frac{F(\zeta)}{\left(\zeta-z_{0}\right)^{3}}\left(\frac{1}{(\zeta-x)^{2}}+\frac{3}{(\zeta-x)\left(x-z_{0}\right)}\right) d \zeta \\
= & \int_{\Sigma \backslash D} \lambda_{0}(\zeta)\left(\frac{\partial}{\partial x} \frac{1}{\zeta-x}+\frac{3}{x-z_{0}} \frac{1}{\zeta-x}\right) d m(\zeta),
\end{aligned}
$$

we have

$$
\begin{gathered}
\frac{f(x)}{\left(x-z_{0}\right)^{3}}-e_{\sigma}^{\prime}(x)-\frac{3 e_{\sigma}(x)}{x-z_{0}} \\
=\sigma \int_{\mathbf{R}} K(\sigma t)\left(\sum_{ \pm} \sum_{l=0}^{4} I_{l}^{ \pm}\right) d t+I_{5},
\end{gathered}
$$

where

$$
\begin{aligned}
& \Sigma_{0}:=\{z \in \mathbf{C} \backslash E: d(z, E)<1\} \\
& I_{0}^{ \pm}:= \int_{\mathbf{H}_{ \pm} \cap \Sigma \backslash \Sigma_{0}} \lambda_{0}(\zeta)\left[\frac{\partial}{\partial x}\left(\frac{1}{\zeta-x}-R^{ \pm}(\zeta, x)\right)\right. \\
&\left.+\frac{3}{x-z_{0}}\left(\frac{1}{\zeta-x}-R^{ \pm}(\zeta, x)\right)\right] d m(\zeta), \\
& I_{1}^{ \pm}:= \int_{\mathbf{H}_{ \pm} \cap \Sigma_{0} \backslash D} \lambda_{0}(\zeta) \frac{\partial}{\partial x}\left(\frac{1}{\zeta-x}-R^{ \pm}(\zeta, x)\right) d m(\zeta), \\
& I_{2}^{ \pm}:=-\int_{\mathbf{H}_{ \pm} \cap D} \lambda_{0}(\zeta) \frac{\partial}{\partial x} R^{ \pm}(\zeta, x) d m(\zeta), \\
& I_{3}^{ \pm}:= \frac{3}{x-z_{0}} \int_{\mathbf{H}_{ \pm} \cap D} \lambda_{0}(\zeta)\left(\frac{1}{\zeta-x}-R^{ \pm}(\zeta, x)\right) d m(\zeta), \\
& I_{4}^{ \pm}:=-\frac{3}{x-z_{0}} \int_{\mathbf{H}_{ \pm} D} \lambda_{0}(\zeta) R^{ \pm}(\zeta, x) d m(\zeta), \\
& I_{5}:= \frac{1}{2 \pi i} \int_{J} \frac{P_{x}(\zeta)-F(\zeta)}{\left(\zeta-z_{0}\right)^{3}}\left(\frac{1}{(\zeta-x)^{2}}+\frac{3}{(\zeta-x)\left(x-z_{0}\right)}\right) d \zeta .
\end{aligned}
$$

According to Lemma 4 for $\zeta \in G_{ \pm}$and $x \in E$,

$$
\begin{aligned}
\left|\frac{\zeta_{\sigma, t}^{ \pm}-\zeta}{\zeta_{\sigma, t}^{ \pm}-x}\right| & =\left|\frac{\zeta_{\sigma}^{ \pm}-\zeta}{\zeta_{\sigma}^{ \pm}-x}\right|\left|\frac{\zeta_{\sigma, t}^{ \pm}-\zeta}{\zeta_{\sigma}^{ \pm}-\zeta}\right|\left|\frac{\zeta_{\sigma}^{ \pm}-x}{\zeta_{\sigma, t}^{ \pm}-x}\right| \\
& \preceq(1+\sigma|t|)^{8}\left|\frac{\zeta_{\sigma}^{ \pm}-\zeta}{\zeta_{\sigma}^{ \pm}-x}\right|,
\end{aligned}
$$




$$
\left|\frac{\zeta_{\sigma}^{ \pm}-x}{\zeta_{\sigma, t}^{ \pm}-x}\right| \preceq(1+\sigma|t|)^{4} .
$$

Since by Lemma 6 for $\zeta \in \mathbf{H}_{ \pm}$and $x \in E$,

$$
|\zeta-x| \preceq\left|\zeta_{\sigma}^{ \pm}-x\right|
$$

and

$$
\begin{aligned}
& \frac{1}{\zeta-x}-R^{ \pm}(\zeta, x)=\frac{1}{\zeta-x}\left(\frac{\zeta_{\sigma, t}^{ \pm}-\zeta}{\zeta_{\sigma, t}^{ \pm}-x}\right)^{9}, \\
& \frac{\partial}{\partial x}\left[\frac{1}{\zeta-x}-R^{ \pm}(\zeta, x)\right] \\
= & \frac{1}{(\zeta-x)^{2}}\left(\frac{\zeta_{\sigma, t}^{ \pm}-\zeta}{\zeta_{\sigma, t}^{ \pm}-x}\right)^{9}+\frac{9}{\zeta-x} \frac{\left(\zeta_{\sigma, t}^{ \pm}-\zeta\right)^{9}}{\left(\zeta_{\sigma, t}^{ \pm}-x\right)^{10}},
\end{aligned}
$$

according to (7.3), (7.4), and Lemma 18 for $\zeta \in \mathbf{H}_{ \pm} \cap \Sigma \backslash D$ we have

$$
\begin{gathered}
\left|\frac{1}{\zeta-x}-R^{ \pm}(\zeta, x)\right| \preceq \frac{1}{|\zeta-x|}\left(\frac{d}{|\zeta-x|}\right)^{9 / 4}(1+\sigma|t|)^{72}, \\
\left|\frac{\partial}{\partial x}\left(\frac{1}{\zeta-x}-R^{ \pm}(\zeta, x)\right)\right| \preceq \frac{1}{|\zeta-x|^{2}}\left(\frac{d}{|\zeta-x|}\right)^{9 / 4}(1+\sigma|t|)^{76} .
\end{gathered}
$$

Moreover, by virtue of Lemmas 4 and 17 for $\zeta \in \mathbf{H}_{ \pm} \cap D$,

$$
\begin{gathered}
\left|R^{ \pm}(\zeta, x)\right| \preceq(1+\sigma|t|)^{68} \sum_{j=1}^{8} \frac{\left|\zeta_{\sigma}^{ \pm}-\zeta\right|^{j}}{\left|\zeta_{\sigma}^{ \pm}-x\right|^{j+1}} \preceq \frac{(1+\sigma|t|)^{68}}{d}, \\
\left|\frac{\partial}{\partial x} R^{ \pm}(\zeta, x)\right| \preceq(1+\sigma|t|)^{72} \sum_{j=1}^{8} \frac{\left|\zeta_{\sigma}^{ \pm}-\zeta\right|^{j}}{\left|\zeta_{\sigma}^{ \pm}-x\right|^{j+2}} \preceq \frac{(1+\sigma|t|)^{72}}{d^{2}} .
\end{gathered}
$$

According to (1.10), (4.36), and Lemma 24(ii) we obtain

$$
\begin{gathered}
\left|\lambda_{0}(\zeta)\right|\left|\zeta-z_{0}\right|^{3} \preceq \\
\begin{cases}\omega\left(\left|\phi_{ \pm}(x)-\phi_{ \pm}(x \pm i|x-\zeta|)\right|\right), & \text { if } \zeta \in \mathbf{H}_{ \pm} \cap \Sigma_{0}, \\
|\zeta||| f||_{C(E)}, & \text { if } \zeta \in \mathbf{H}_{ \pm} \cap \Sigma \backslash \Sigma_{0} .\end{cases}
\end{gathered}
$$

Next, applying (7.5)-(7.9) we estimate each of the integrals

$$
\tilde{I}_{l}^{ \pm}:=\left|\sigma \int_{\mathbf{R}} K(\sigma t) I_{l}^{ \pm} d t\right| \quad(l=0, \ldots, 4)
$$


from above.

Since by (4.21) and Lemma 21

$$
d \preceq \sigma^{-1}
$$

and

$$
\tilde{I}_{0}^{ \pm} \preceq \frac{\|f\|_{C(E)}}{\sigma^{9 / 4}} \int_{\mathbf{H}_{ \pm} \cap \Sigma \backslash \Sigma_{0}}\left(\frac{1}{|\zeta-x|}+\frac{1}{\left|x-z_{0}\right|}\right) \frac{d m(\zeta)}{\left|\zeta-z_{0}\right|^{2}|\zeta-x|^{13 / 4}}
$$

dividing the set of integration into subsets

$$
\begin{aligned}
& U_{1}^{ \pm}:=\mathbf{H}_{ \pm} \cap\left(\Sigma \backslash \Sigma_{0}\right) \cap D\left(x, \frac{1}{2}\left|x-z_{0}\right|\right), \\
& U_{2}^{ \pm}:=\mathbf{H}_{ \pm} \cap\left(\Sigma \backslash \Sigma_{0}\right) \cap D\left(x, 2\left|x-z_{0}\right|\right) \backslash U_{1}^{ \pm}, \\
& U_{3}^{ \pm}:=\mathbf{H}_{ \pm} \cap\left(\Sigma \backslash \Sigma_{0}\right) \backslash\left(U_{1}^{ \pm} \cup U_{2}^{ \pm}\right),
\end{aligned}
$$

and passing to the polar coordinates with the center either in $x$ or in $z_{0}$ we obtain

$$
\tilde{I}_{0}^{ \pm} \preceq \frac{\|f\|_{C(E)}}{\sigma\left|x-z_{0}\right|^{3}} .
$$

To deal with $\tilde{I}_{1}^{ \pm}$, we note that by virtue of (1.10), (4.22), and (4.23)

$$
\frac{\omega\left(\left|\phi_{ \pm}(x)-\phi_{ \pm}(x \pm i|x-\zeta|)\right|\right)}{\omega\left(\left|\phi_{ \pm}(x)-\phi_{ \pm}(x \pm i d)\right|\right)} \preceq\left|\frac{\phi_{ \pm}(x)-\phi_{ \pm}(x \pm i|x-\zeta|)}{\phi_{ \pm}(x)-\phi_{ \pm}(x \pm i d)}\right| \preceq \frac{|x-\zeta|}{d} .
$$

Therefore, according to (1.10) and (2.19)

$$
\tilde{I}_{1}^{ \pm} \preceq \omega\left(\frac{1}{\sigma}\right) d^{5 / 4} \int_{\mathbf{H}_{ \pm} \cap \Sigma_{0} \backslash D} \frac{d m(\zeta)}{\left|\zeta-z_{0}\right|^{3}|\zeta-x|^{13 / 4}} .
$$

Next, dividing the set of integration $\mathbf{H}_{ \pm} \cap \Sigma_{0} \backslash D$ into subsets

$$
\begin{aligned}
& V_{1}^{ \pm}:=\mathbf{H}_{ \pm} \cap\left(\Sigma_{0} \backslash D\right) \cap D\left(x, \frac{1}{2}\left|x-z_{0}\right|\right), \\
& V_{2}^{ \pm}:=\mathbf{H}_{ \pm} \cap\left(\Sigma_{0} \backslash D\right) \cap D\left(x, 2\left|x-z_{0}\right|\right) \backslash V_{1}^{ \pm}, \\
& V_{3}^{ \pm}:=\mathbf{H}_{ \pm} \cap\left(\Sigma_{0} \backslash D\right) \backslash\left(V_{1}^{ \pm} \cup V_{2}^{ \pm}\right)
\end{aligned}
$$

and passing to the polar coordinates with the center either in $x$ or in $z_{0}$ as well as applying (7.10) we obtain

$$
\tilde{I}_{1}^{ \pm} \preceq \omega\left(\frac{1}{\sigma}\right)\left|x-z_{0}\right|^{-3}
$$


In the same manner we can prove that

$$
\tilde{I}_{3}^{ \pm} \preceq \omega\left(\frac{1}{\sigma}\right)\left|x-z_{0}\right|^{-3} .
$$

In order to estimate $\tilde{I}_{2}^{ \pm}$and $\tilde{I}_{4}^{ \pm}$we note that by (2.19) and (4.13) for $\zeta \in \mathbf{H}_{ \pm} \cap D$,

$$
\begin{gathered}
\left|\phi_{ \pm}(x)-\phi_{ \pm}(x \pm i|x-\zeta|)\right| \preceq \frac{1}{\sigma}, \\
\left|\zeta_{\sigma}^{ \pm}-\zeta\right| \succeq d .
\end{gathered}
$$

Therefore,

$$
\begin{aligned}
& \tilde{I}_{2}^{ \pm} \preceq \omega\left(\frac{1}{\sigma}\right)\left|x-z_{0}\right|^{-3} d^{-2} \int_{\mathbf{H}_{ \pm} \cap D} d m(\zeta) \preceq \omega\left(\frac{1}{\sigma}\right)\left|x-z_{0}\right|^{-3}, \\
& \tilde{I}_{4}^{ \pm} \preceq \omega\left(\frac{1}{\sigma}\right)\left|x-z_{0}\right|^{-4} d^{-1} \int_{\mathbf{H}_{ \pm} \cap D} d m(\zeta) \preceq \omega\left(\frac{1}{\sigma}\right)\left|x-z_{0}\right|^{-3} .
\end{aligned}
$$

Furthermore, (4.36) and Lemma 24(iii) imply that

$$
\begin{aligned}
\left|I_{5}\right| & \preceq \omega\left(\frac{1}{\sigma}\right)\left|x-z_{0}\right|^{-3} d \int_{J}\left(\frac{1}{|\zeta-x|^{2}}+\frac{1}{|\zeta-x|\left|x-z_{0}\right|}\right)|d \zeta| \\
& \preceq \omega\left(\frac{1}{\sigma}\right)\left|x-z_{0}\right|^{-3} .
\end{aligned}
$$

Comparing (7.2) and (7.11)-(7.16) we have (7.1).

\section{Proof of Theorem 3}

We adapt to our case the standard procedure of proving inverse theorems. Let $x_{1}, x_{2} \in E$ be such that $x_{1}<x_{2}$ and let $\delta:=\rho_{E}\left(x_{1}, x_{2}\right)$. Since

$$
\left|f\left(x_{2}\right)-f\left(x_{1}\right)\right| \leq 2|| f \|_{C(E)},
$$

we can assume that $0<\delta<1 / 2$.

Let $e_{k} \in E_{2^{k}}, k \in \mathbf{N}_{0}$ satisfy

$$
\left\|f-e_{k}\right\|_{C(E)} \leq 2 \omega\left(2^{-k}\right)
$$

and let

$$
g_{k}(z):=e_{k+1}(z)-e_{k}(z) .
$$


Chose $m \in \mathbf{N}$ such that

$$
2^{-m-1}<\delta \leq 2^{-m}
$$

Since

$$
\begin{aligned}
f\left(x_{2}\right)-f\left(x_{1}\right) & =\left[f\left(x_{2}\right)-e_{m}\left(x_{2}\right)\right]-\left[f\left(x_{1}\right)-e_{m}\left(x_{1}\right)\right] \\
& +e_{0}\left(x_{2}\right)-e_{0}\left(x_{1}\right)+\sum_{k=0}^{m-1}\left[g_{k}\left(x_{2}\right)-g_{k}\left(x_{1}\right)\right],
\end{aligned}
$$

we have

$$
\left|f\left(x_{2}\right)-f\left(x_{1}\right)\right| \leq 4 \omega(2 \delta)+\int_{x_{1}}^{x_{2}}\left|e_{0}^{\prime}(x)\right| d x+\sum_{k=0}^{m-1} \int_{x_{1}}^{x_{2}}\left|g_{k}^{\prime}(x)\right| d x .
$$

Furthermore, since for $x \in E$ and $k=0, \ldots, m-1$,

$$
\begin{gathered}
\left|e_{0}(x)\right| \leq|f(x)|+\left|f(x)-e_{0}(x)\right| \leq\|f\|_{C(E)}+2 \omega(1), \\
\left|g_{k}(x)\right| \leq\left|e_{k+1}(x)-f(x)\right|+\left|f(x)-e_{k}(x)\right| \leq 4 \omega\left(2^{-k}\right),
\end{gathered}
$$

by the Phragmen-Lindelöf theorem (see [13, VIIIA]) for $z \in \mathbf{C}$,

$$
\begin{gathered}
\left|e_{0}(z)\right| \leq\left(\|f\|_{C(E)}+2 \omega(1)\right) \exp \left(C_{1}|\Im \phi(z)|\right), \\
\left|g_{k}(z)\right| \leq 4 \omega\left(2^{-k}\right) \exp \left(C_{1} 2^{k+1}|\Im \phi(z)|\right),
\end{gathered}
$$

where $\phi$ is the Levin conformal mapping extended to $\mathbf{H}_{-}$by the formula

$$
\phi(z):=\overline{\phi(\bar{z})} \quad\left(z \in \mathbf{H}_{-}\right) .
$$

Let for $x \in E$,

$$
E_{\delta}^{ \pm}:=\left\{z \in \overline{\mathbf{H}}_{ \pm}: \pm \Im \phi(z)=\delta\right), \quad \rho_{\delta}(x)=d\left(x, E_{\delta}^{ \pm}\right) .
$$

According to (3.5) for $x \in E$ and $0<t<T$,

$$
\left(\frac{t}{T}\right)^{C_{2}} \preceq \frac{\rho_{t}(x)}{\rho_{T}(x)} \preceq \frac{t}{T} .
$$

Since by (1.7)

$$
x_{2}-x_{1}<C_{3} \delta<\frac{C_{3}}{2}
$$

and according to (3.4) and (8.4)

$$
2\left(x_{2}-x_{1}\right) \leq \rho_{C \delta}\left(x_{1}\right),
$$


by virtue of (1.10), (3.1), and (8.2)-(8.4) for $x_{1} \leq x \leq x_{2}$ we have

$$
\begin{aligned}
& \left|e_{0}^{\prime}(x)\right| \leq \frac{1}{2 \pi} \int_{\left|z-x_{1}\right|=C_{3}} \frac{\left|e_{0}(z)\right|}{|z-x|^{2}}|d z| \preceq\|f\|_{C(E)}+\frac{\omega(\delta)}{\delta}, \\
& \left|g_{k}^{\prime}(x)\right| \leq \frac{1}{2 \pi} \int_{\left|z-x_{1}\right|=\rho_{C 2^{-k}}\left(x_{1}\right)} \frac{\left|g_{k}(z)\right|}{|z-x|^{2}}|d z| \preceq \frac{\omega\left(2^{-k}\right)}{\rho_{2^{-k}}\left(x_{1}\right)} .
\end{aligned}
$$

Therefore,

$$
\begin{aligned}
& \int_{x_{1}}^{x_{2}}\left|e_{0}^{\prime}(x)\right| d x \preceq \delta\|f\|_{C(E)}+\omega(\delta), \\
& \sum_{k=0}^{m-1} \int_{x_{1}}^{x_{2}}\left|g_{k}^{\prime}(x)\right| d x \preceq \sum_{k=0}^{m-1} \frac{\omega\left(2^{-k}\right) \rho_{2^{-m}}\left(x_{1}\right)}{\rho_{2^{-k}}\left(x_{1}\right)} \\
& \preceq \sum_{k=0}^{m-1} 2^{k-m} \omega\left(2^{-k}\right) \preceq \delta \int_{\delta}^{1} \frac{\omega(t)}{t^{2}} d t .
\end{aligned}
$$

Comparing (8.1), (8.5), and (8.6) we obtain (1.12).

\section{Acknowledgements}

This research was conducted while the author was visiting the Katholische Universität Eichstaett and Universität Trier. The author wishes to thank the members of these universities for the pleasant mathematical atmosphere they offered him. The author would also like to warmly thank Misha Nesterenko for many useful remarks.

\section{References}

[1] N. I. Achieser (1992): Theory of Approximation. New York: Dover Publications.

[2] L. V. Ahlfors (1973): Conformal Invariants, Topics in Geometric Function Theory. New York: McGraw-Hill.

[3] V. V. Andrievskii (1986): The geometric structure of regions, and direct theorems of the constructive theory of functions. Math. USSR-Sb., 54:39-56. 
[4] V. V. Andrievskii (1989): Metric properties of Riemann's mapping function for the region supplemented to continuum without external zero angles. Sov. J. Contemp. Math. Anal., Arm. Acad. Sci., 24:57-68.

[5] V. V. Andrievskii (2003): On Belyi's lemma. East J. on Approx., 9:111-115.

[6] V. V. Andrievskii (2006): Positive harmonic functions on Denjoy domains in the complex plane. Preprint 06-20, GSF Neuherberg, Institute of Biomathematics and Biometry.

[7] V. V. Andrievskii, V. I. Belyi, V. K. Dzjadyk (1995): Conformal Invariants in Constructive Theory of Functions of Complex Variable. Atlanta, GA: World Federation.

[8] Z. Ditzian, V. Totik (1987): Moduli of smoothness. New York, Springer Verlag.

[9] E. M. Dyn'kin (1977): On the uniform approximation of functions in Jordan domains. Sibirsk. Mat. Zh., 18:775-786. (Russian).

[10] V. K. Dzjadyk (1977): Introduction to the Theory of Uniform Approximation of Functions by Polynomials. Moskow: Nauka (Russian).

[11] J. B. Garnett, D. E. Marshall (2005): Harmonic Measure. Cambridge: Cambridge University Press.

[12] J. A. Jenkins, K. Oikawa (1971): On results of Ahlfors and Hayman. Illinois J. Math., 15:664-671.

[13] P. Koosis (1988): The Logarithmic Integral, I. Cambridge: Cambridge University Press.

[14] O. Lehto, K. I. Virtanen (1973): Quasiconformal Mappings in the Plane, 2nd ed.. New York: Springer-Verlag.

[15] B. Ya. Levin (1989): Majorants in classes of subharmonic functions. I-III, in Teor. Funktsii, Funktsional. Anal. i Prilozhen., 51:3-17; 52:3-33 (Russian).

[16] B. Ya. Levin (1993): Completeness of systems of functions, quasianalyticity and subharmonic majorants. J. Soviet Math., 63:171-201.

[17] Ch. Pommerenke (1975): Univalent Functions. Göttingen: Vandenhoeck \& Ruprecht.

[18] N. A. Shirokov (2003): Approximation by entire functions on an infinite system of closed intervals. Amer. Mat. Soc. Transl., 209:183-190. 
[19] N. A. Shirokov (2004): Inverse approximation theorem on an infinite union of segments. Journal of Math. Sci., 124:4935-4939.

[20] E. M. Stein (1970): Singular Integrals and Differentiability Properties of Functions. Princeton, NJ: Princeton University Press.

[21] P. M. Tamrazov (1975): Smoothnesses and Polynomial Approximations. Kiev: Naukova Dumka (Russian).

[22] A. F. Timan (1994): Theory of Approximation of Functions of a Real Variable, New York: Dover Publications.

V. V. Andrievskii

Department of Mathematical Sciences

Kent State University

Kent, OH 44242

USA

e-mail: andriyev@math.kent.edu 